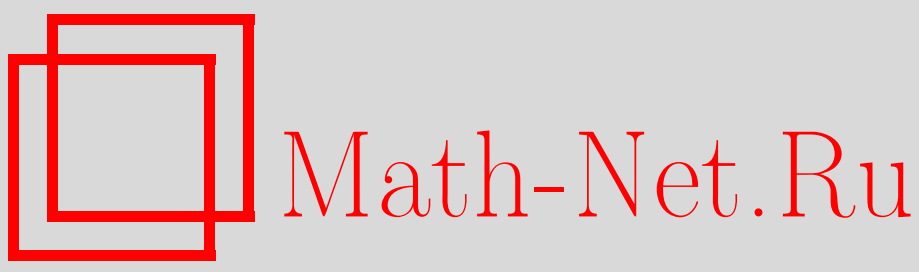

С. Г. Танкеев, О группе Брауэра арифметической схемы, Изв. РАН. Сер. матем., 2001, том 65, выпуск 2, 155-186

DOI: https://doi.org/10.4213/im330

Использование Общероссийского математического портала Math-Net.Ru подразумевает, что вы прочитали и согласны с пользовательским соглашением

http: //www.mathnet.ru/rus/agreement

Параметры загрузки:

IP : 54.157 .27 .8

26 апреля 2023 г., $05: 41: 44$ 
УДК 512.6

\author{
С.Г. Танкеев
}

\title{
О группе Брауэра арифметической схемы
}

\begin{abstract}
Для поверхности Энриквеса $V$ над числовым полем $k$ с $k$-рациональной точкой доказывается, что $l$-компонента $\operatorname{Br}(V) / \operatorname{Br}(k)$ конечна, если и только если $l \neq 2$. Для регулярного проективного гладкого многообразия, удовлетворяющего гипотезе Тэйта для дивизоров над числовым полем, находится простой критерий конечности $l$-компоненты $\mathrm{Br}^{\prime}(V) / \mathrm{Br}(k)$. Более того, для арифметической модели $X$ многообразия $V$ доказывается вариант гипотезы Артина о конечности группы Брауэра $X$. Даются приложения к вопросу о конечности $l$-компонент групп Шаффаревича-Тэйта.

Библиография: 21 наименование.
\end{abstract}

\section{Введение}

Пусть $V$ - такое гладкое проективное гиперкэлерово многообразие над числовым полем $k$, что $V(k) \neq \varnothing$ и число Бетти $\mathrm{b}_{2}(V \otimes \bar{k})>3$. Тогда для любого простого числа $l$-примарная компонента групшы $\operatorname{Br}^{\prime}(V) / \operatorname{Br}(k)$ конечна [19, теорема 3.3]. Более того, предположим, что $k$ - чисто мнимое числовое поле и существует собственный плоский морфизм $\pi: X \rightarrow \operatorname{Spec}(A)$, где $A$ - кольцо целых поля $k, X$ - регулярная схема, обший слой $\pi$ изоморфен $V$, все схемные слои морфизма $\pi$ приведены, сушествует такое сечение $\theta: \operatorname{Spec}(A) \rightarrow X$ морфизма $\pi$, что $\theta(\operatorname{Spec}(A))$ пересекается в точности с одной неприводимой компонентой любого схемного слоя $\pi^{-1}(v)$, $v \in \operatorname{Spec}(A)$ (мы называем $\pi: X \rightarrow \operatorname{Spec}(A)$ арифметической моделью $V$ ). Тогда для любого простого числа $l l$-примарная компонента группы $\operatorname{Br}^{\prime}(X)$ конечна $[19$, следствие 6.2].

В этой статье мы исследуем вопрос о конечности $l$-компонент $\operatorname{Br}(V) / \operatorname{Br}(k)$ и $\operatorname{Br}^{\prime}(X)$ для поверхности Энриквеса $V$ над числовым полем $k$.

Напомним, что геометрически минимальная модель $V$ поверхности Энриквеса характеризуется как гладкая проективная поверхность с $H^{1}\left(V, \mathscr{O}_{V}\right)=(0)$, $H^{2}\left(V, \mathscr{O}_{V}\right)=(0),\left(\Omega_{V}^{2}\right)^{\otimes 2} \simeq \mathscr{O}_{V}$.

ТЕОРЕМА 0.1. Пусть $V$ - гладкая проективная геометрически минимальная поверхность Энриквеса над числовым полем $k$. Предположим, что $V(k) \neq \varnothing u l$ - простое число. Тогда l-примарная компонента группь $\operatorname{Br}(V) / \operatorname{Br}(k)$ конечна, если и только если $l \neq 2$.

Работа выполнена при финансовой поддержке Центра грантов Новосибирского государственного университета, при частичной поддержке DFG-гранта Forschergruppe Arithmetik (университеты Маннгейма и Гейдельберга, апрель 1999). Я хотел бы поблагодарить оба университета за теплое гостеприимство и превосходные условия для работы.

$$
\text { (C) С.Г. ТАнкеEв, } 2001
$$


ЗАмЕчАниЕ. Для гладкой проективной поверхности $S$ над конечным полем $\mathbb{F}_{q}$ конечность $l$-компоненты $\operatorname{Br}(S) \simeq \operatorname{Br}(S) / \operatorname{Br}\left(\mathbb{F}_{q}\right)$ для одного простого числа $l \neq$ $\operatorname{char}\left(\mathbb{F}_{q}\right)$ влечет конечность $l$-компоненты $\operatorname{Br}(S)$ для любого простого числа $l \neq$ $\operatorname{char}\left(\mathbb{F}_{q}\right)\left[21\right.$, теорема 5.2]. Более того, если $\operatorname{char}\left(\mathbb{F}_{q}\right) \neq 2$, то конечность $l$-компонент $\operatorname{Br}(S) \simeq \operatorname{Br}(S) / \operatorname{Br}\left(\mathbb{F}_{q}\right)$ для одного простого числа (включая случай $l=\operatorname{char}\left(\mathbb{F}_{q}\right)$ ) влечет конечность $l$-компоненты $\operatorname{Br}(S)$ для любого простого числа $l$ [10, теорема 4.1]. Теорема 0.1 показывает, что для поверхности Энриквеса $V$ над числовым полем ситуация существенно отличается от ситуации над конечным полем.

Для гладкого проективного многообразия $V$ над числовым полем $k$ и для простого числа $l$ рассмотрим $l$-адическое представление в этальных когомологиях

$$
\rho_{l}: \operatorname{Gal}(\bar{k} / k) \rightarrow \operatorname{GL}\left(H^{2}\left(V \otimes \bar{k}, \mathbb{Q}_{l}(1)\right)\right) .
$$

Дж. Тэйт предположил, что

$$
\mathrm{NS}(V \otimes \bar{k}) \otimes \mathbb{Q}_{l} \simeq H^{2}\left(V \otimes \bar{k}, \mathbb{Q}_{l}(1)\right)^{\operatorname{Lie} \operatorname{Im}\left(\rho_{l}\right)}
$$

[20, гипотеза 1]. Эта гипотеза влечет соотношение

$$
\mathrm{NS}(V) \otimes \mathbb{Q}_{l} \simeq H^{2}\left(V \otimes \bar{k}, \mathbb{Q}_{l}(1)\right)^{\operatorname{Gal}(\bar{k} / k)},
$$

где (по определению $[21, \S 4]) \mathrm{NS}(V)$ является образом $\operatorname{Pic}(V)$ в $\mathrm{NS}(V \otimes \bar{k})$. Ecли (0.1) выполняется для всех достаточно больших конечных расширений поля $k$, то гипотеза Тэйта верна.

Следующий результат является естественным обобщением теоремы 0.1 на случай регулярного гладкого проективного многообразия, удовлетворяющего гипотезе Тэйта.

ТЕОрема 0.2. Пусть $V$ - гладкое проективное многообразие над числовым полем $k$. Предположим, что

$$
\begin{gathered}
V(k) \neq \varnothing, \quad H^{1}\left(V \otimes \bar{k}, \mathscr{O}_{V \otimes \bar{k}}\right)=(0), \quad \operatorname{NS}(V)=\mathrm{NS}(V \otimes \bar{k}), \\
\mathrm{NS}(V) \otimes \mathbb{Q}_{l} \widetilde{\rightarrow}\left[H^{2}\left(V \otimes \bar{k}, \mathbb{Q}_{l}(1)\right)\right]^{\operatorname{Gal}(\bar{k} / k)}
\end{gathered}
$$

для простого числа l и для многообразия $V$.

Тогда l-примарная компонента группь $\operatorname{Br}^{\prime}(V) / \operatorname{Br}(k)$ конечна, если и только если l не делит $\operatorname{Card}\left([\mathrm{NS}(V \otimes \bar{k})]_{\text {tors }}\right)$.

Следуюшая теорема является модификацией теоремы 6.1 из [19] и дает вариант гипотезы M. Артина [11, гл. IV,$\S 2$, вопрос 2.19] о конечности группы Брауэра арифметической собственной схемы над $\operatorname{Spec}(\mathbb{Z})$.

Теорема 0.3. Пусть $V$ - такое гладжое проективное многообразие над числовым полем $k$, ито $V(k) \neq \varnothing, H^{1}\left(V \otimes \bar{k}, \mathscr{O}_{V \otimes \bar{k}}\right)=(0)$,

$$
\mathrm{NS}(V) \otimes \mathbb{Q}_{l} \widetilde{\rightarrow}\left[H^{2}\left(V \otimes \bar{k}, \mathbb{Q}_{l}(1)\right)\right]^{\operatorname{Gal}(\bar{k} / k)}
$$

для некоторого простого числа l. Предположим существование собственного плоского морфизма $\pi: X \rightarrow \operatorname{Spec}(A)$, где $A-$ кольцо иелых поля $k, X-$ регулярная схема, общий слой $\pi$ изоморфен $V$, все схемнье слои морфизма $\pi$ 
приведень, существует такое сечение $\theta: \operatorname{Spec}(A) \rightarrow X$ морфизма $\pi$, что $\theta(\operatorname{Spec}(A))$ пересекается в точности с одной неприводимой компонентой любого схемного слоя $\pi^{-1}(v), v \in \operatorname{Spec}(A)$ (мы называем $\pi: X \rightarrow \operatorname{Spec}(A)$ арифметической моделью $V)$.

Ecли l не делит $\operatorname{Card}\left([\mathrm{NS}(V \otimes \bar{k})]_{\mathrm{tors}}\right)$, то l-примарная компонента группьь $\mathrm{Br}^{\prime}(X)$ конечна.

Следуюший результат дает интересные соотношения между группами Брауэра геометрической и арифметической моделей многообразия $V$ над чисто трансцендентным расширением числового поля.

ТеОрема 0.4. Пусть $V$ - гладжое проективное многообразие над $k(t)$, где $k(t)$ - поле рациональных функиий алгебраически независимой переменной $t$ над числовым полем $k \hookrightarrow \mathbb{C},[k: \mathbb{Q}]<\infty, V(k(t)) \neq \varnothing$, и пусть $\pi: X \rightarrow \mathbb{P}_{k}^{1}-$ такой собственный плоский $k$-морфизм гладких проективных $k$-многообразий, что общий слой $\pi$ изоморфен $V$ (мы называем $\pi: X \rightarrow \mathbb{P}_{k}^{1}$ геометрической моделью $V$ ).

Тогда существует естественное вложение

$$
\mathrm{Br}^{\prime}(X) / \operatorname{Br}(k) \hookrightarrow \mathrm{Br}^{\prime}(V) / \operatorname{Br}(k(t))
$$

Eсли $H^{1}\left(V \otimes \overline{k(t)}, \mathscr{O}_{V \otimes \overline{k(t)}}\right)=(0), \mathrm{NS}(V \otimes \overline{k(t)})=\mathrm{NS}(V)$, nростое число $l$ не делит $\operatorname{Card}\left([\mathrm{NS}(V \otimes \overline{k(t)})]_{\text {tors }}\right) u$

$$
\mathrm{NS}(V) \otimes \mathbb{Q}_{l} \widetilde{\rightarrow}\left[H^{2}\left(V \otimes \overline{k(t)}, \mathbb{Q}_{l}(1)\right)\right]^{\mathrm{Gal}(\overline{k(t)} / k(t))}
$$

то l-примарные компоненты групп $\operatorname{Br}^{\prime}(X) / \operatorname{Br}(k)$ u $\operatorname{Br}^{\prime}(V) / \operatorname{Br}(k(t))$ конечны. Более того, в рассматриваемом случае предположим существование соб-

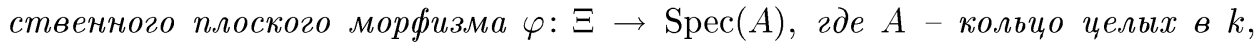
$\Xi$ - регулярная схема, обший слой $\varphi$ изоморфен $X$, все схемные слои морфизма $\varphi$ приведены, существует такое сечение $\theta: \operatorname{Spec}(A) \rightarrow \Xi$ морфизма $\varphi$, что $\theta(\operatorname{Spec}(A))$ пересекается в точности с одной неприводимой компонентой любого схемного слоя $\varphi^{-1}(v), v \in \operatorname{Spec}(A)$ (мы называем $\varphi: \Xi \rightarrow \operatorname{Spec}(A)$ арифметической моделью $X)$; тогда l-компонента группь $\mathrm{Br}^{\prime}(\Xi)$ конечна.

Следующая теорема показывает, что при некоторых предположениях можно вывести гипотезу Тэйта для регулярного многообразия $X$ над числовым полем $k$ из гипотезы Тэйта для общего слоя $V$ сюръективного $k$-морфизма $X \rightarrow \mathbb{P}_{k}^{1}$.

ТЕОрема 0.5. Пусть $V$ - гладкое проективное многообразие над $k(t)$, где $k(t)$ - поле рациональных функиий алгебраически независимой переменной $t$ над иисловым полем $k \hookrightarrow \mathbb{C},[k: \mathbb{Q}]<\infty$, и пусть $\pi: X \rightarrow \mathbb{P}_{k}^{1}-$ такой собственный плоский $k$-морфизм гладких проективных $k$-многообразий, что общий слой $\pi$ изоморфен $V$ (мы называем $\pi: X \rightarrow \mathbb{P}_{k}^{1}$ геометрической моделью $\left.V\right)$.

Eсли $H^{1}\left(V \otimes \overline{k(t)}, \mathscr{O}_{V \otimes \overline{k(t)}}\right)=(0), \mathrm{NS}(V \otimes \overline{k(t)})=\mathrm{NS}(V), V(k(t)) \neq \varnothing$, $\mathrm{NS}(X \otimes \bar{k})=\mathrm{NS}(X)$, простое число $l$ не делит $\operatorname{Card}\left([\mathrm{NS}(V \otimes \overline{k(t)})]_{\text {tors }}\right) u$ $\operatorname{Card}\left([\mathrm{NS}(X \otimes \bar{k})]_{\text {tors }}\right)$, канонический морфизм

$$
\operatorname{Br}^{\prime}(X)(l) \rightarrow\left[\operatorname{Br}^{\prime}(X \otimes \bar{k})(l)\right]^{\operatorname{Gal}(\bar{k} / k)}
$$


сюргективен и

$$
\mathrm{NS}(V) \otimes \mathbb{Q}_{l} \underset{\rightarrow}{\sim}\left[H^{2}\left(V \otimes \overline{k(t)}, \mathbb{Q}_{l}(1)\right)\right]^{\mathrm{Gal}(\overline{k(t)} / k(t))}
$$

то существует канонический изоморфизм

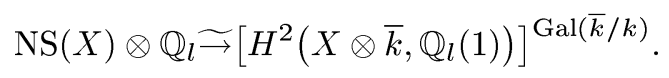

Пусть $C$ - регулярная превосходная неприводимая 1 -мерная схема, $K$ - поле рациональных функций на $C$. Пусть $\widehat{K}_{v}$ - пополнение $K$ относительно нормирования, определенного замкнутой точкой $v \in C$. Множество всех таких пополнений используется в определении группы Шафаревича-Тэйта абелева многообразия $J$ над $K$ :

$$
\operatorname{III}(C, J)=\operatorname{Ker}\left[H^{1}(\operatorname{Spec}(K), J) \rightarrow \prod_{\operatorname{codim}_{C}(v)=1} H^{1}\left(\operatorname{Spec}\left(\widehat{K}_{v}\right), J\right)\right] .
$$

Пусть $K_{v}^{\mathrm{h}}\left(\right.$ соответственно $\left.K_{v}^{\mathrm{sh}}\right)$ - поле частных обьчной гензелизации $\mathscr{O}_{C, v}^{\mathrm{h}}($ соответственно строгой гензелизации $\left.\mathscr{O}_{C, v}^{\mathrm{sh}}=\mathscr{O}_{C, \bar{v}}\right)$ локального кольца $\mathscr{O}_{C, v}$. Мы определяем "гензелеву" и “строго гензелеву" модификации групшы Шафаревича-Тэйта следующими формулами:

$$
\begin{gathered}
\operatorname{III}^{\mathrm{h}}(C, J)=\operatorname{Ker}\left[H^{1}(\operatorname{Spec}(K), J) \rightarrow \prod_{\operatorname{codim}_{C}(v)=1} H^{1}\left(\operatorname{Spec}\left(K_{v}^{\mathrm{h}}\right), J\right)\right], \\
\mathrm{III}^{\mathrm{sh}}(C, J)=\operatorname{Ker}\left[H^{1}(\operatorname{Spec}(K), J) \rightarrow \prod_{\operatorname{codim}_{C}(v)=1} H^{1}\left(\operatorname{Spec}\left(K_{v}^{\mathrm{sh}}\right), J\right)\right] .
\end{gathered}
$$

Имеем

$$
\mathrm{III}(C, J)=\operatorname{III}^{\mathrm{h}}(C, J) \hookrightarrow \mathrm{III}^{\mathrm{sh}}(C, J) .
$$

ТеОРема 0.6. Пусть $C$ - такая регулярная превосходная нётерова 1-мерная неприводимая схема, что поле вычетов любой замкнутой точки $v \in C$ конечно, $K$ - поле рациональных функций на $C$. Тогда для абелева многообразия $J$ над $K \quad \operatorname{III}(C, J)=\operatorname{III}^{\mathrm{h}}(C, J) \hookrightarrow \operatorname{III}^{\mathrm{sh}}(C, J)$ является подгруппой конечного индекса. Более того, если геометрический слой минимальной модели Нерона многообразия $J$ в любой точке $v \in C$ связен (например, если $J$ имеет хорошую редукиию в каждой точке $v \in C), \operatorname{mo} \operatorname{III}(C, J)=\operatorname{III}^{\mathrm{h}}(C, J)=$ $\operatorname{III}^{\mathrm{sh}}(C, J)$.

ТЕОРема 0.7. Пусть $C$ - гладкая проективная кривая над числовым полем $k, V$ - гладкая проективная поверхность над $k$, и пусть $f: V \rightarrow C-$ $k$-морфизм с 1-мерными приведенными и неприводимыми геометрическими слоями. Предположим, что выполняются следующие условия:

а) существует сечение $\theta: C \rightarrow V$ морфизма $f, C(k) \neq \varnothing$, общий слой $V_{\eta}$ морфизма $f$ гладкий;

b) сечение $\theta$ определяет изоморфизм $\operatorname{Pic}^{0}(C) \simeq \operatorname{Pic}^{0}(V)$.

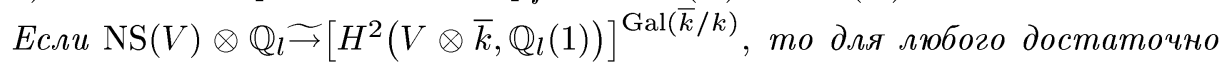
больиого простого числа l l-примарные компоненты групп $\operatorname{III}^{\mathrm{sh}}\left(C, \operatorname{Pic}^{0}\left(V_{\eta}\right)\right)$ $u \operatorname{III}\left(C, \operatorname{Pic}^{0}\left(V_{\eta}\right)\right)$ конечны. 
СлЕДСТВИЕ 0.8. Пусть $V$ - гладкая проективная поверхность над иисловым полем $k$, и пусть $f: V^{\prime} \rightarrow \mathbb{P}_{k}^{1}-$ такой пучок Лефшеца сечений $V$ гиперповерхностями степени $d \geqslant 3$, что любой замкнутый слой геометрически приведен и неприводим с не более чем одной обыкновенной двойной особой точкой. $М$ оь обозначаем через $V_{\eta}^{\prime}$ общий слой $f$.

Если $H^{1}\left(V, \mathscr{O}_{V}\right)=(0)$ u $\mathrm{NS}(V) \otimes \mathbb{Q}_{l} \widetilde{\rightarrow}\left[H^{2}\left(V \otimes \bar{k}, \mathbb{Q}_{l}(1)\right)\right]^{\operatorname{Gal}(\bar{k} / k)}$, то для любого достаточно большого простого числа l l-примарные компоненты әрупп $\operatorname{III}^{\mathrm{sh}}\left(\mathbb{P}_{k}^{1}, \operatorname{Pic}^{0}\left(V_{\eta}^{\prime}\right)\right)$ и $\operatorname{III}\left(\mathbb{P}_{k}^{1}, \operatorname{Pic}^{0}\left(V_{\eta}^{\prime}\right)\right)$ конечны.

Напомним, что имеется список регулярных поверхностей, удовлетворяющих гипотезе Тэйта: рациональная поверхность, поверхность Энриквеса [19, лемма 3.2], поверхность типа К3 [17], поверхность Ферма [19, §3.3].

Автор благодарен Р. Пинку за возможность издать препринт [18], содержаший первоначальную версию этой статьи, и за теплое гостеприимство во время моего пребывания в университете Маннгейма.

\section{§1. О группе Брауэра поверхности Энриквеса}

1.1. Лемма. Пусть $k$ - числовое поле. Тогда для любого простого числа $p$ $\operatorname{Hom}_{\text {cont }}(\operatorname{Gal}(\bar{k} / k), \mathbb{Z} / p \mathbb{Z})$ является бесконечной группой.

ДокАЗАТЕльСтво. Пусть $S$ - такое конечное множество неархимедовых точек $k$, что $\operatorname{char}(k(v)) \equiv 1(\bmod p)$ для любой точки $v \in S$. Если $l=\operatorname{char} k(v)$, то для некоторого натурального числа $f$ мы имеем $\operatorname{Norm}_{k / \mathbb{Q}}(v)=l^{f} ;$ более того, поскольку $l \equiv 1(\bmod p)$, мы получаем соотношение $\operatorname{Norm}_{k / \mathbb{Q}}(v) \equiv 1(\bmod p)$.

Следуя работам $\mathrm{X}$. Коха, обозначим через $k_{S}$ максимальное $p$-расширение поля $k$, неразветвленное вне $S$. Пусть $k_{v}$ - пополнение $k$, определенное дискретным нормированием $v$. Для данного поля $L$ пусть

$$
\delta(L)= \begin{cases}1, & \text { если } L \text { содержит корень из единицы степени } p, \\ 0 & \text { в противоположном случае. }\end{cases}
$$

Рассмотрим $\mathbb{Z} / p \mathbb{Z}$ с тривиальным действием $\operatorname{Gal}\left(k_{S} / k\right)$. Пусть $E$ - группа всех единиц $k$. Мы имеем

$$
\begin{gathered}
\operatorname{dim}_{\mathbb{Z} / p \mathbb{Z}} \operatorname{Hom}_{\operatorname{cont}}\left(\operatorname{Gal}\left(k_{S} / k\right), \mathbb{Z} / p \mathbb{Z}\right)=\operatorname{dim}_{\mathbb{Z} / p \mathbb{Z}} H^{1}\left(\operatorname{Gal}\left(k_{S} / k\right), \mathbb{Z} / p \mathbb{Z}\right) \\
=\sum_{\substack{v \in S \\
\operatorname{char}(k(v))=p}}\left[k_{v}: \mathbb{Q}_{p}\right]-\delta(k)-r+1+\sum_{v \in S} \delta\left(k_{v}\right)+\operatorname{dim}_{\mathbb{Z} / p \mathbb{Z}} B_{S},
\end{gathered}
$$

где $r$ - число всех архимедовых точек $k$, и

$$
0 \leqslant \operatorname{dim}_{\mathbb{Z} / p \mathbb{Z}} B_{S} \leqslant \operatorname{dim}_{\mathbb{Z} / p \mathbb{Z}} \mathrm{Cl}(k)_{p}+\operatorname{dim}_{\mathbb{Z} / p \mathbb{Z}} E / E^{p}
$$

$[9$, предложения $11.8,11.7]$. В силу хорошо известной теоремы Дирихле множество $\{l \mid l$-простое число,$l \equiv 1(\bmod p)\}$ бесконечно [15, гл. VI, $\S 4$, теорема 2$]$. Поэтому множество

$$
T=\{v \mid v \text {-неархимедова точка } k, \operatorname{char}(k(v)) \equiv 1(\bmod p)\}
$$


бесконечно. Заметим, что $S \subset T$.

Для любого $v \in T$ имеем $\delta\left(k_{v}\right)=1$. Действительно, $l=\operatorname{char}(k(v))$, поэтомy $p|(l-1)| \operatorname{Card}\left(k(v)^{\times}\right)$; в силу леммы Гензеля поле $k_{v}$ содержит примитивньй корень из единицы степени $\operatorname{Card}\left(k(v)^{\times}\right)$[4, гл. II, $\left.\S 7\right]$; в частности, $k_{v}$ содержит корень из единицы степени $p$. Поэтому (1.1) имеет вид

$$
\begin{aligned}
& \operatorname{dim}_{\mathbb{Z} / p \mathbb{Z}} \operatorname{Hom}_{\operatorname{cont}}\left(\operatorname{Gal}\left(k_{S} / k\right), \mathbb{Z} / p \mathbb{Z}\right)= \\
& \quad=\sum_{\substack{v \in S \\
\operatorname{char}(k(v))=p}}\left[k_{v}: \mathbb{Q}_{p}\right]-\delta(k)-r+1+\operatorname{Card}(S)+\operatorname{dim}_{\mathbb{Z} / p \mathbb{Z}} B_{S} .
\end{aligned}
$$

Для любого натурального числа $N$ можно выбрать $S=S(N)$ настолько большим, что

$$
\operatorname{dim}_{\mathbb{Z} / p \mathbb{Z}} \operatorname{Hom}_{\text {cont }}\left(\operatorname{Gal}\left(k_{S} / k\right), \mathbb{Z} / p \mathbb{Z}\right) \geqslant N .
$$

Это утверждение следует из (1.2) и из бесконечности множества $T$. С другой стороны, естественный сюръективный морфизм $\operatorname{Gal}(\bar{k} / k) \rightarrow \operatorname{Gal}\left(k_{S} / k\right)$ дает точную последовательность

$$
0 \rightarrow \operatorname{Hom}_{\text {cont }}\left(\operatorname{Gal}\left(k_{S} / k\right), \mathbb{Z} / p \mathbb{Z}\right) \rightarrow \operatorname{Hom}_{\text {cont }}(\operatorname{Gal}(\bar{k} / k), \mathbb{Z} / p \mathbb{Z}),
$$

являющуюся началом канонической точной последовательности инфлящии - ограничения

$$
0 \rightarrow H^{1}\left(\operatorname{Gal}\left(k_{S} / k\right), \mathbb{Z} / p \mathbb{Z}\right) \stackrel{\inf }{\rightarrow} H^{1}(\operatorname{Gal}(\bar{k} / k), \mathbb{Z} / p \mathbb{Z}) \stackrel{\text { res }}{\rightarrow} H^{1}\left(\operatorname{Gal}\left(\bar{k} / k_{S}\right), \mathbb{Z} / p \mathbb{Z}\right)
$$

$\left[4\right.$, гл. IV,$\S 5$, предложение 5.1]. Поэтому $\operatorname{dim}_{\mathbb{Z} / p \mathbb{Z}} \operatorname{Hom}_{\text {cont }}(\operatorname{Gal}(\bar{k} / k), \mathbb{Z} / p \mathbb{Z}) \geqslant N$ для любого натурального числа $N$. Лемма доказана.

1.2. В этом пункте мы доказываем теорему 0.1 .

Напомним, что существует точная последовательность

$$
0 \rightarrow\left(\mathrm{NS}(V \otimes \bar{k})_{\mathrm{tors}}\right)^{*} \rightarrow \pi_{1}(V \otimes \bar{k})^{\mathrm{ab}} \rightarrow \prod_{l} T_{l}(\operatorname{Alb}(V \otimes \bar{k})) \rightarrow 0,
$$

где $\left(\mathrm{NS}(V \otimes \bar{k})_{\text {tors }}\right)^{*}$ - группа, двойственная по Понтрягину к конечной группе $\mathrm{NS}(V \otimes \bar{k})_{\text {tors }}\left[11\right.$, гл. III, $\S 4$, следствие 4.19]. Равенство $H^{1}\left(V, \mathscr{O}_{V}\right)=(0)$ показывает, что $\operatorname{Alb}(V \otimes \bar{k})=(0)$. С другой стороны, поверхность Энриквеса $V \otimes \bar{k}$ является фактором односвязной поверхности типа К3 по инволюции без неподвижных точек $[6$, гл. $4, \S 5]$. В частности, $\pi_{1}(V \otimes \bar{k}) \simeq \mathbb{Z} / 2 \mathbb{Z}$. Следовательно,

$$
\operatorname{Pic}(V \otimes \bar{k})_{\text {tors }}=\mathrm{NS}(V \otimes \bar{k})_{\text {tors }} \simeq \mathbb{Z} / 2 \mathbb{Z}
$$

имеет каноническую образующую $\Omega_{V}^{2}$, определенную над $k$. Поэтому $\operatorname{Gal}(\bar{k} / k)$ действует тривиально на $\mathrm{NS}(V \otimes \bar{k})_{\text {tors }}$.

Если $l \neq 2$, то $l>\operatorname{Card}\left([\operatorname{NS}(V \otimes \bar{k})]_{\text {tors }}\right) ;$ поскольку $H^{2}\left(V, \mathscr{O}_{V}\right)=(0)$, то мы имеем равенство $\operatorname{rank} \mathrm{NS}\left(V \otimes_{k} \bar{k}\right)=h^{1,1}\left(V \otimes_{k} \bar{k}\right)$. В силу теоремы 3.3 из [19] $l$-примарная компонента группы $\operatorname{Br}(V) / \operatorname{Br}(k)$ конечна. 
Предположим, что $l=2$. Тогда мы имеем точную последовательность 2-примарных компонент [19, точная последовательность (2.14)]

$$
\begin{aligned}
0 & \rightarrow \operatorname{Br}(k)(2) \rightarrow \operatorname{Ker}\left[\operatorname{Br}(V)(2) \rightarrow \operatorname{Br}(V \otimes \bar{k})(2)^{\operatorname{Gal}(\bar{k} / k)}\right] \\
& \rightarrow H^{1}(\operatorname{Gal}(\bar{k} / k), \operatorname{NS}(V \otimes \bar{k}))(2) \rightarrow 0 .
\end{aligned}
$$

Рассмотрим точную последовательность $\mathrm{Gal}(\bar{k} / k)$-модулей

$$
0 \rightarrow \mathrm{NS}(V \otimes \bar{k})_{\text {tors }} \rightarrow \mathrm{NS}(V \otimes \bar{k}) \rightarrow \mathrm{NS}(V \otimes \bar{k}) / \mathrm{NS}(V \otimes \bar{k})_{\text {tors }} \rightarrow 0 .
$$

Она дает точную последовательность

$$
\begin{aligned}
{\left[\mathrm{NS}(V \otimes \bar{k}) / \mathrm{NS}(V \otimes \bar{k})_{\mathrm{tors}}\right]_{\mathrm{Gal}(\bar{k} / k)} } & \rightarrow H^{1}\left(\operatorname{Gal}(\bar{k} / k), \mathrm{NS}(V \otimes \bar{k})_{\mathrm{tors}}\right) \\
& \rightarrow H^{1}(\operatorname{Gal}(\bar{k} / k), \mathrm{NS}(V \otimes \bar{k})) .
\end{aligned}
$$

Заметим, что $H^{1}\left(\operatorname{Gal}(\bar{k} / k), \mathrm{NS}(V \otimes \bar{k})_{\text {tors }}\right)$ аннулируется $2=\operatorname{Card}\left(\mathrm{NS}(V \otimes \bar{k})_{\text {tors }}\right)$, поэтому

$$
\operatorname{Im}\left[\left[\mathrm{NS}(V \otimes \bar{k}) / \mathrm{NS}(V \otimes \bar{k})_{\mathrm{tors}}\right]^{\mathrm{Gal}(\bar{k} / k)} \rightarrow H^{1}\left(\mathrm{Gal}(\bar{k} / k), \mathrm{NS}(V \otimes \bar{k})_{\mathrm{tors}}\right)\right]
$$

является конечной 2-группой.

В силу (1.3) и (1.4) остается показать, что $H^{1}\left(\mathrm{Gal}(\bar{k} / k), \mathrm{NS}(V \otimes \bar{k})_{\text {tors }}\right)$ - бесконечная 2-группа. Напомним, что $\operatorname{Gal}(\bar{k} / k)$ действует тривиально на $\operatorname{NS}(V \otimes$ $\bar{k})_{\text {tors }} \simeq \mathbb{Z} / 2 \mathbb{Z}$. Поэтому

$$
H^{1}\left(\operatorname{Gal}(\bar{k} / k), \mathrm{NS}(V \otimes \bar{k})_{\text {tors }}\right) \simeq \operatorname{Hom}_{\text {cont }}(\operatorname{Gal}(\bar{k} / k), \mathbb{Z} / 2 \mathbb{Z}),
$$

и теорема 0.1 следует из леммы 1.1 .

\section{§2. Гипотеза Тэйта и группа Брауэра регулярного многообразия}

2.1. В этом пункте мы доказываем теорему 0.2 .

Хорошо известно, что $H^{1}\left(V \otimes \bar{k}, \mathscr{O}_{V \otimes \bar{k}}\right)=(0)$ - касательное пространство в нуле группы $\operatorname{Pic}^{0}(V \otimes \bar{k})$ [13, лекция 24]. Поэтому $\operatorname{Pic}^{0}(V \otimes \bar{k})=(0)$. Следовательно,

$$
\operatorname{Pic}(V \otimes \bar{k}) \simeq \operatorname{Pic}(V \otimes \bar{k}) / \operatorname{Pic}^{0}(V \otimes \bar{k})=\mathrm{NS}(V \otimes \bar{k}),
$$

и точная последовательность (2.11) из [19] имеет вид

$$
\begin{aligned}
0 & \rightarrow \operatorname{Br}(k) \rightarrow \operatorname{Ker}\left[\operatorname{Br}^{\prime}(V) \rightarrow \operatorname{Br}^{\prime}(V \otimes \bar{k})^{\operatorname{Gal}(\bar{k} / k)}\right] \\
& \rightarrow H^{1}(\operatorname{Gal}(\bar{k} / k), \operatorname{NS}(V \otimes \bar{k})) \rightarrow 0 .
\end{aligned}
$$

Поскольку $\mathrm{NS}(V)=\mathrm{NS}(V \otimes \bar{k})$, то группа $\operatorname{Gal}(\bar{k} / k)$ действует тривиально на $\mathrm{NS}(V \otimes \bar{k}) \simeq \mathbb{Z}^{\rho} \oplus[\mathrm{NS}(V \otimes \bar{k})]_{\text {tors }}$ (неканонический изоморфизм, где выполнено равенство $\rho=\operatorname{rank} \mathrm{NS}(V \otimes \bar{k}))$. Поэтому

$$
\begin{aligned}
& H^{1}(\operatorname{Gal}(\bar{k} / k), \operatorname{NS}(V \otimes \bar{k}))=\operatorname{Hom}_{\text {cont }}(\operatorname{Gal}(\bar{k} / k), \operatorname{NS}(V \otimes \bar{k})) \\
& \quad=\operatorname{Hom}_{\mathrm{cont}}\left(\operatorname{Gal}(\bar{k} / k), \mathbb{Z}^{\rho}\right) \oplus \operatorname{Hom}_{\text {cont }}\left(\operatorname{Gal}(\bar{k} / k),[\operatorname{NS}(V \otimes \bar{k})]_{\text {tors }}\right) \\
& \quad=\operatorname{Hom}_{\mathrm{cont}}\left(\operatorname{Gal}(\bar{k} / k),[\mathrm{NS}(V \otimes \bar{k})]_{\mathrm{tors}}\right),
\end{aligned}
$$


так как $\mathbb{Z}^{\rho}$ не содержит нетривиальных компактных подгрупп, и в результате мы имеем $\operatorname{Hom}_{\text {cont }}\left(\operatorname{Gal}(\bar{k} / k), \mathbb{Z}^{\rho}\right)=(0)$.

Следовательно, точная последовательность (2.1) имеет вид

$$
\begin{aligned}
0 & \rightarrow \operatorname{Br}(k) \rightarrow \operatorname{Ker}\left[\operatorname{Br}^{\prime}(V) \rightarrow \operatorname{Br}^{\prime}(V \otimes \bar{k})^{\operatorname{Gal}(\bar{k} / k)}\right] \\
& \rightarrow H^{1}\left(\operatorname{Gal}(\bar{k} / k),[\mathrm{NS}(V \otimes \bar{k})]_{\text {tors }}\right) \rightarrow 0 .
\end{aligned}
$$

Все группы в (2.2) являются группами кручения, поэтому мы получаем точную последовательность $l$-примарных компонент

$$
\begin{aligned}
0 & \rightarrow \operatorname{Br}(k)(l) \rightarrow \operatorname{Ker}\left[\operatorname{Br}^{\prime}(V)(l) \rightarrow \operatorname{Br}^{\prime}(V \otimes \bar{k})(l)^{\operatorname{Gal}(\bar{k} / k)}\right] \\
& \rightarrow H^{1}\left(\operatorname{Gal}(\bar{k} / k),[\mathrm{NS}(V \otimes \bar{k})]_{\text {tors }}\right)(l) \rightarrow 0
\end{aligned}
$$

Заметим, что $H^{1}\left(\operatorname{Gal}(\bar{k} / k),[\operatorname{NS}(V \otimes \bar{k})]_{\text {tors }}\right)=\operatorname{Hom}_{\text {cont }}\left(\operatorname{Gal}(\bar{k} / k),[\operatorname{NS}(V \otimes \bar{k})]_{\text {tors }}\right)$ аннулируется числом $\operatorname{Card}\left([\mathrm{NS}(V \otimes \bar{k})]_{\mathrm{tors}}\right)$.

Если простое число $l$ не делит $\operatorname{Card}\left([\mathrm{NS}(V \otimes \bar{k})]_{\mathrm{tors}}\right)$, то подгруппа $l$-кручения в $H^{1}\left(\mathrm{Gal}(\bar{k} / k),[\mathrm{NS}(V \otimes \bar{k})]_{\mathrm{tors}}\right)$ тривиальна, и $(2.3)$ дает изоморфизм

$$
\operatorname{Br}(k)(l) \simeq \operatorname{Ker}\left[\operatorname{Br}^{\prime}(V)(l) \rightarrow \operatorname{Br}^{\prime}(V \otimes \bar{k})(l)^{\operatorname{Gal}(\bar{k} / k)}\right],
$$

поэтому утверждение следует из конечности $\operatorname{Br}^{\prime}(V \otimes \bar{k})(l)^{\mathrm{Gal}(\bar{k} / k)}[19$, теорема 2.4].

Если простое число $l$ делит $\operatorname{Card}\left([\mathrm{NS}(V \otimes \bar{k})]_{\mathrm{tors}}\right)$, то подгруппа $l$-кручения в $[\mathrm{NS}(V \otimes \bar{k})]_{\text {tors }}$ нетривиальна, поэтому она содержит подгруппу $E \simeq \mathbb{Z} / l \mathbb{Z}$.

Рассмотрим точную последовательность $\mathrm{Gal}(\bar{k} / k)$-модулей

$$
0 \rightarrow E \rightarrow \mathrm{NS}(V \otimes \bar{k})_{\text {tors }} \rightarrow \mathrm{NS}(V \otimes \bar{k})_{\text {tors }} / E \rightarrow 0
$$

с тривиальным действием $\mathrm{Gal}(\bar{k} / k)$. Она дает точную последовательность

$$
\begin{aligned}
{\left[\mathrm{NS}(V \otimes \bar{k})_{\mathrm{tors}} / E\right]^{\operatorname{Gal}(\bar{k} / k)} } & \rightarrow H^{1}(\operatorname{Gal}(\bar{k} / k), E) \\
& \rightarrow H^{1}\left(\operatorname{Gal}(\bar{k} / k), \mathrm{NS}(V \otimes \bar{k})_{\mathrm{tors}}\right) .
\end{aligned}
$$

Заметим, что $H^{1}(\operatorname{Gal}(\bar{k} / k), E)$ аннулируется числом $l=\operatorname{Card}(E)$, поэтому

$$
\operatorname{Im}\left[\left[\operatorname{NS}(V \otimes \bar{k})_{\mathrm{tors}} / E\right]^{\operatorname{Gal}(\bar{k} / k)} \rightarrow H^{1}(\operatorname{Gal}(\bar{k} / k), E)\right]
$$

является конечной $l$-группой. В силу $(2.3)$ остается показать, что $H^{1}(\operatorname{Gal}(\bar{k} / k), E)$ - бесконечная $l$-группа.

Поскольку $\operatorname{Gal}(\bar{k} / k)$ действует тривиально на $E \simeq \mathbb{Z} / l \mathbb{Z}$, мы имеем соотношение

$$
H^{1}(\operatorname{Gal}(\bar{k} / k), E) \simeq \operatorname{Hom}_{\text {cont }}(\operatorname{Gal}(\bar{k} / k), \mathbb{Z} / l \mathbb{Z}),
$$

поэтому теорема 0.2 следует из леммы 1.1 . 
2.2. В этом пункте мы доказываем теорему 0.3 .

Пусть $i_{y}: \operatorname{Spec}(k(y)) \rightarrow X-$ каноническое вложение $y \in X$ и

$$
\operatorname{Div}_{X}^{\text {vert }}=\bigoplus_{\substack{y \in X \backslash V \\ \operatorname{codim}_{X}(y)=1}} i_{y^{*}} \mathbb{Z}
$$

- пучок "вертикальных" дивизоров Картье.

Существует точная последовательность пучков (в этальной топологии $X$ )

$$
0 \rightarrow \mathrm{G}_{m, X} \rightarrow h_{*} \mathrm{G}_{m, V} \rightarrow \operatorname{Div}_{X}^{\text {vert }} \rightarrow 0
$$

где $h: V \rightarrow X-$ вложение обшего слоя $\pi[19,(6.1)]$. Она дает точную последовательность

$$
0 \rightarrow \operatorname{Br}^{\prime}(X) \rightarrow \operatorname{Ker}\left[\operatorname{Br}^{\prime}(V) \rightarrow H^{0}\left(X, R^{2} h_{*} \mathrm{G}_{m, V}\right)\right] \rightarrow H^{2}\left(X, \operatorname{Div}_{X}^{\text {vert }}\right)
$$

(см. $[19,(6.4)])$.

Начиная с этого места мы обозначаем через $k(y)^{s}$ сепарабельное замыкание $k(y)$ в $\overline{k(y)}$. Поскольку $V(k) \neq \varnothing$, то сушествует каноническое вложение $\operatorname{Br}(k) \hookrightarrow$ $\mathrm{Br}^{\prime}(V)$. С другой стороны, существует каноническая точная последовательность

$$
\begin{aligned}
0 & \rightarrow \operatorname{Br}(A) \rightarrow \operatorname{Br}(k) \rightarrow \bigoplus_{v \text { неарx }} \operatorname{Hom}_{\text {cont }}\left(\operatorname{Gal}\left(k(v)^{s} / k(v)\right), \mathbb{Q} / \mathbb{Z}\right) \\
& \rightarrow H^{3}\left(\operatorname{Spec}(A), \mathrm{G}_{m}\right) \rightarrow H^{3}\left(\operatorname{Spec}(k), \mathrm{G}_{m}\right) \rightarrow 0
\end{aligned}
$$

$[11$, гл. III, $\S 3$, пример $2.22(\mathrm{e})]$. В силу глобальной теории полей классов существует точная последовательность

$$
0 \rightarrow \operatorname{Br}(k) \rightarrow\left[\bigoplus_{v \text { Heapx }} \operatorname{Br}\left(k_{v}\right)\right] \oplus\left[\bigoplus_{v \text { apx }} \operatorname{Br}\left(k_{v}\right)\right] \stackrel{\Sigma}{\rightarrow} \mathbb{Q} / \mathbb{Z} \rightarrow 0
$$

где $k_{v}$ - пополнение $k$, определенное точкой $v$.

Рассмотрим каноническую проекцию

$$
\operatorname{pr}_{\text {apx }}:\left[\bigoplus_{v \text { Heapx }} \operatorname{Br}\left(k_{v}\right)\right] \oplus\left[\bigoplus_{v \text { apx }} \operatorname{Br}\left(k_{v}\right)\right] \rightarrow\left[\bigoplus_{v \text { apx }} \operatorname{Br}\left(k_{v}\right)\right],
$$

и пусть

$$
\operatorname{Br}(k)^{\text {неарх }}=\operatorname{Br}(k) \cap \operatorname{Ker}\left(\operatorname{pr}_{\text {apx }}\right) .
$$

Поскольку $\left[\bigoplus_{v \text { aрх }} \operatorname{Br}\left(k_{v}\right)\right]$ - конечная группа, мы видим, что $\operatorname{Br}(k)^{\text {неарх }} \hookrightarrow \operatorname{Br}(k)-$ подгруппа конечного индекса, и сушествует каноническое вложение

$$
\operatorname{Br}(k)^{\text {неарх }} \hookrightarrow\left[\bigoplus_{v \text { неарх }} \operatorname{Br}\left(k_{v}\right)\right] .
$$


Используя существование коммутативных диаграмм [11, гл. III, $\S 3$, пример 2.22 , (c), (f)] для всех неархимедовых точек $v$ :

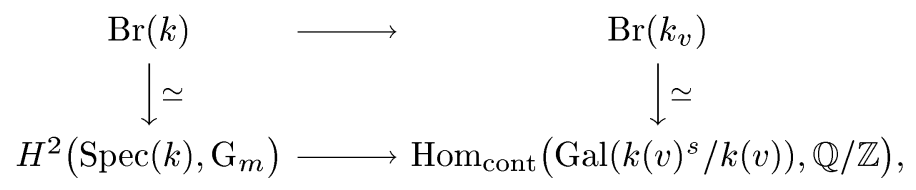

мы перепишем (2.5) как

$$
0 \rightarrow \operatorname{Br}(k)^{\text {неapx }} \rightarrow \underset{\begin{array}{c}
v \in \operatorname{Spec}(A) \\
\operatorname{codim} \operatorname{Spec}(A)
\end{array}(v)=1}{ } \operatorname{Hom}_{\text {cont }}\left(\operatorname{Gal}\left(k(v)^{s} / k(v)\right), \mathbb{Q} / \mathbb{Z}\right) .
$$

Рассмотрим каноническое вложение $i_{v}: \operatorname{Spec}(k(v)) \hookrightarrow \operatorname{Spec}(A)$. Спектральная последовательность Лере

$$
E_{2}^{p, q}=H^{p}\left(\operatorname{Spec}(A), R^{q} i_{v^{*}} \mathbb{Z}\right) \Longrightarrow H^{p+q}(\operatorname{Spec}(k(v)), \mathbb{Z})
$$

дает точную последовательность

$$
0 \rightarrow E_{2}^{1,0} \rightarrow E^{1} \rightarrow E_{2}^{0,1} \stackrel{d_{2}^{0,1}}{\rightarrow} E_{2}^{2,0} \rightarrow E_{1}^{2} \rightarrow E_{2}^{1,1},
$$

где $E_{1}^{2}=\operatorname{Ker}\left[E^{2} \rightarrow E_{2}^{0,2}\right][11$, приложение В]; следовательно, мы имеем точную последовательность

$$
\begin{aligned}
0 & \rightarrow H^{1}\left(\operatorname{Spec}(A), i_{v^{*}} \mathbb{Z}\right) \rightarrow H^{1}(\operatorname{Spec}(k(v)), \mathbb{Z}) \rightarrow H^{0}\left(\operatorname{Spec}(A), R^{1} i_{v^{*}} \mathbb{Z}\right) \\
& \stackrel{d_{2}^{0,1}}{\rightarrow} H^{2}\left(\operatorname{Spec}(A), i_{v^{*}} \mathbb{Z}\right) \rightarrow \operatorname{Ker}\left[H^{2}(\operatorname{Spec}(k(v)), \mathbb{Z})\right. \\
& \left.\rightarrow H^{0}\left(\operatorname{Spec}(A), R^{2} i_{v^{*}} \mathbb{Z}\right)\right] \rightarrow H^{1}\left(\operatorname{Spec}(A), R^{1} i_{v^{*}} \mathbb{Z}\right) .
\end{aligned}
$$

Известно, что $R^{q} i_{v^{*}} \mathbb{Z}=(0)$ для всех $q>0$ [11, гл. III, $\S 2$, пример $\left.2.22(\mathrm{a})\right]$; поэтому (2.7) дает изоморфизм

$$
H^{2}\left(\operatorname{Spec}(A), i_{v^{*}} \mathbb{Z}\right) \simeq H^{2}(\operatorname{Spec}(k(v)), \mathbb{Z}) ;
$$

с другой стороны, $H^{2}(\operatorname{Spec}(k(v)), \mathbb{Z}) \simeq \operatorname{Hom}_{\text {cont }}\left(\operatorname{Gal}\left(k(v)^{s} / k(v)\right), \mathbb{Q} / \mathbb{Z}\right)[11$, гл. III, $\S 2$, пример 2.22]. Следовательно,

$$
H^{2}\left(\operatorname{Spec}(A), \operatorname{Div}_{\operatorname{Spec}(A)}\right)=\bigoplus_{\substack{v \in \operatorname{Spec}(A) \\ \operatorname{codim}_{\operatorname{Spec}(A)}(v)=1}} \operatorname{Hom}_{\operatorname{cont}}\left(\operatorname{Gal}\left(k(v)^{s} / k(v)\right), \mathbb{Q} / \mathbb{Z}\right)
$$

[11, гл. III, §2, пример $2.22(\mathrm{a})]$. Поэтому (2.6) имеет вид

$$
0 \rightarrow \operatorname{Br}(k)^{\text {неарх }} \rightarrow H^{2}\left(\operatorname{Spec}(A), \operatorname{Div}_{\operatorname{Spec}(A)}\right) .
$$

Пусть $\theta: \operatorname{Spec}(A) \rightarrow X$ - сечение морфизма $\pi$, удовлетворяющее условиям теоремы. Оно определяет инъективные морфизмы

$$
\pi^{*}: \operatorname{Br}(k) \hookrightarrow \operatorname{Br}^{\prime}(V), \quad H^{2}\left(\operatorname{Spec}(A), \operatorname{Div}_{\operatorname{Spec}(A)}\right) \hookrightarrow H^{2}\left(X, \operatorname{Div}_{X}^{\text {vert }}\right)
$$


$[19,(6.12)]$. Пусть $B=\operatorname{Ker}\left[\operatorname{Br}^{\prime}(V) \rightarrow H^{0}\left(X, R^{2} h_{*} \mathrm{G}_{m, V}\right)\right]$. Очевидно, что (2.4) и (2.8) дают коммутативную диаграмму с точными строками

$$
\begin{aligned}
& 0 \longrightarrow \mathrm{Br}^{\prime}(X) \longrightarrow \quad \mathrm{B} \quad \longrightarrow \quad H^{2}\left(X, \mathrm{Div}_{X}^{\text {vert }}\right) \\
& \cup \quad U \\
& 0 \longrightarrow \operatorname{Br}(k)^{\text {неарх }} \cap B \longrightarrow H^{2}\left(\operatorname{Spec}(A), \operatorname{Div}_{\operatorname{Spec}(A)}\right) .
\end{aligned}
$$

Следовательно, $\operatorname{Br}^{\prime}(X) \cap \operatorname{Br}(k)^{\text {неарх }}=(0)$ внутри $\operatorname{Br}^{\prime}(V)$. Поэтому

$$
\operatorname{Br}^{\prime}(X) \hookrightarrow \operatorname{Br}^{\prime}(V) / \operatorname{Br}(k)^{\text {неарх }} ;
$$

с другой стороны, существует точная последовательность

$$
0 \rightarrow \operatorname{Br}(k) / \mathrm{Br}(k)^{\text {неарх }} \rightarrow \mathrm{Br}^{\prime}(V) / \mathrm{Br}(k)^{\text {неарх }} \rightarrow \mathrm{Br}^{\prime}(V) / \mathrm{Br}(k) \rightarrow 0
$$

c конечной группой $\operatorname{Br}(k) / \operatorname{Br}(k)^{\text {неарх }}$. Остается показать, что $l$-примарная компонента группь $\mathrm{Br}^{\prime}(V) / \mathrm{Br}(k)$ конечна.

Хорошо известно, что $H^{1}\left(V \otimes \bar{k}, \mathscr{O}_{V \otimes \bar{k}}\right)=(0)$ - касательное пространство в нуле групшы $\operatorname{Pic}^{0}(V \otimes \bar{k})\left[13\right.$, лекция 24]. Поэтому $\operatorname{Pic}^{0}(V \otimes \bar{k})=(0)$. Следовательно,

$$
\operatorname{Pic}(V \otimes \bar{k}) \simeq \operatorname{Pic}(V \otimes \bar{k}) / \operatorname{Pic}^{0}(V \otimes \bar{k})=\mathrm{NS}(V \otimes \bar{k}),
$$

и поэтому (2.11) из [19] имеет вид

$$
\begin{aligned}
0 & \rightarrow \operatorname{Br}(k) \rightarrow \operatorname{Ker}\left[\operatorname{Br}^{\prime}(V) \rightarrow \operatorname{Br}^{\prime}(V \otimes \bar{k})^{\operatorname{Gal}(\bar{k} / k)}\right] \\
& \rightarrow H^{1}(\operatorname{Gal}(\bar{k} / k), \mathrm{NS}(V \otimes \bar{k})) \rightarrow 0 .
\end{aligned}
$$

В силу предложения 2.3 из [19] все группы в (2.9) являются группами кручения, поэтому мы получаем точную последовательность $l$-примарных компонент

$$
\begin{aligned}
0 & \rightarrow \operatorname{Br}(k)(l) \rightarrow \operatorname{Ker}\left[\operatorname{Br}^{\prime}(V)(l) \rightarrow \operatorname{Br}^{\prime}(V \otimes \bar{k})(l)^{\operatorname{Gal}(\bar{k} / k)}\right] \\
& \rightarrow H^{1}(\operatorname{Gal}(\bar{k} / k), \operatorname{NS}(V \otimes \bar{k}))(l) \rightarrow 0
\end{aligned}
$$

$\mathrm{C}$ другой стороны, $\mathrm{NS}(V \otimes \bar{k})$ имеет конечное множество образуюших, и любая образуюшая "определена" над конечным расширением, поэтому мы можем выбрать такое конечное расширение Галуа $K / k$, что $\mathrm{NS}(V \otimes K)=\mathrm{NS}(V \otimes \bar{k})$. Более того, $H^{1}(\mathrm{Gal}(K / k), \mathrm{NS}(V \otimes K))$ - конечная группа [4, гл. IV, $\S 6$, следствие 2].

Рассмотрим каноническую точную последовательность "инфлящии-ограничения" [4, гл. IV,$\S 5$, предложение 5.1$]$

$$
\begin{aligned}
0 & \rightarrow H^{1}(\mathrm{Gal}(K / k), \mathrm{NS}(V \otimes K)) \stackrel{\inf }{\rightarrow} H^{1}(\operatorname{Gal}(\bar{k} / k), \mathrm{NS}(V \otimes \bar{k})) \\
& \stackrel{\operatorname{res}}{\rightarrow} H^{1}(\operatorname{Gal}(\bar{k} / K), \mathrm{NS}(V \otimes \bar{k})) .
\end{aligned}
$$

Поскольку $\mathrm{NS}(V \otimes K)=\mathrm{NS}(V \otimes \bar{k})$, то группа $\operatorname{Gal}(\bar{k} / K)$ действует тривиально на $\mathrm{NS}(V \otimes \bar{k}) \simeq \mathbb{Z}^{\rho} \oplus[\mathrm{NS}(V \otimes \bar{k})]_{\text {tors }}$ (неканонический изоморфизм, где $\rho=$ $\operatorname{rank} \operatorname{NS}(V \otimes \bar{k}))$. Следовательно,

$$
\begin{aligned}
& H^{1}(\operatorname{Gal}(\bar{k} / K), \mathrm{NS}(V \otimes \bar{k}))=\operatorname{Hom}_{\text {cont }}(\operatorname{Gal}(\bar{k} / K), \mathrm{NS}(V \otimes \bar{k})) \\
& \quad=\operatorname{Hom}_{\text {cont }}\left(\operatorname{Gal}(\bar{k} / K), \mathbb{Z}^{\rho}\right) \oplus \operatorname{Hom}_{\text {cont }}\left(\operatorname{Gal}(\bar{k} / K),[\mathrm{NS}(V \otimes \bar{k})]_{\text {tors }}\right) \\
& \quad=\operatorname{Hom}_{\text {cont }}\left(\operatorname{Gal}(\bar{k} / K),[\mathrm{NS}(V \otimes \bar{k})]_{\text {tors }}\right),
\end{aligned}
$$


так как $\mathbb{Z}^{\rho}$ не содержит нетривиальных компактных подгрупп, и в итоге мы имеем $\operatorname{Hom}_{\text {cont }}\left(\operatorname{Gal}(\bar{k} / K), \mathbb{Z}^{\rho}\right)=(0)$.

Следовательно,

$$
H^{1}(\operatorname{Gal}(\bar{k} / K), \operatorname{NS}(V \otimes \bar{k}))=\operatorname{Hom}_{\text {cont }}\left(\operatorname{Gal}(\bar{k} / K),[\operatorname{NS}(V \otimes \bar{k})]_{\text {tors }}\right)
$$

аннулируется числом $\operatorname{Card}\left([\mathrm{NS}(V \otimes \bar{k})]_{\text {tors }}\right)$. Поскольку простое число $l$ не делит $\operatorname{Card}\left([\mathrm{NS}(V \otimes \bar{k})]_{\text {tors }}\right)$, то подгруппа $l$-кручения в $H^{1}(\operatorname{Gal}(\bar{k} / K), \operatorname{NS}(V \otimes \bar{k}))$ тривиальна (иначе эта группа содержит элемент порядка $l$, которьй аннулируется числом $\operatorname{Card}\left([\mathrm{NS}(V \otimes \bar{k})]_{\text {tors }}\right)$, поэтому он аннулируется 1 (наибольшим общим делителем $l$ и $\left.\left.\operatorname{Card}\left([\mathrm{NS}(V \otimes \bar{k})]_{\text {tors }}\right)\right)\right)$.

Поэтому (2.11) и конечность $H^{1}(\operatorname{Gal}(K / k), \mathrm{NS}(V \otimes K))$ показывают, что группа

$$
H^{1}(\operatorname{Gal}(\bar{k} / k), \mathrm{NS}(V \otimes \bar{k}))(l)=H^{1}(\operatorname{Gal}(K / k), \operatorname{NS}(V \otimes K))(l)
$$

конечна.

С другой стороны, соотношения

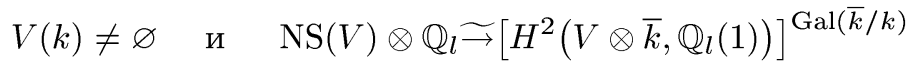

дают конечность $\left[\operatorname{Br}^{\prime}(V \otimes \bar{k})(l)\right]^{\operatorname{Gal}(\bar{k} / k)}[19$, теорема 2.4]. В частности,

$$
\operatorname{Ker}\left[\operatorname{Br}^{\prime}(V)(l) \rightarrow \operatorname{Br}^{\prime}(V \otimes \bar{k})(l)^{\operatorname{Gal}(\bar{k} / k)}\right]
$$

- подгруппа конечного индекса в $\operatorname{Br}^{\prime}(V)(l)$. Следовательно, мы получаем из (2.10) и (2.12) конечность группы $\operatorname{Br}^{\prime}(V)(l) / \operatorname{Br}(k)(l)=\left[\operatorname{Br}^{\prime}(V) / \operatorname{Br}(k)\right](l)$. Tеорема 0.3 доказана.

2.3. Пусть $K$ - поле алгебраических функций одной переменной над числовым полем $k \hookrightarrow \mathbb{C},[k: \mathbb{Q}]<\infty$, и $V$ - геометрически неприводимая гладкая проективная поверхность над $K$. Обозначим через $S$ полную гладкую модель поля $K$ над $k$.

По определению $V$ имеет стабильные редукции во всех точках поля $K$, если и только если существует такой собственный морфизм $\pi: X \rightarrow S$ гладких связных $k$-схем, что его обший схемный слой изоморфен $V$ и выполнены следуюшие условия:

а) любой геометрический слой $X_{\bar{s}}$ приведен;

b) $X_{\bar{s}}$ является объединением гладких неприводимых компонент с нормальными пересечениями на $X \otimes_{k} \bar{k}$.

Согласно хорошо известной теореме о стабильной редукции любая гладкая проективная поверхность над $K$ имеет стабильные редукции во всех точках некоторого конечного алгебраического расширения $K$.

Напомним, что поверхность $V$ имеет стабильное сильное вырожсдене $[16, \S 1]$ в точке $v$ поля $K$, если и только если $V$ имеет стабильную редукцию в точке $v$ и для любой неприводимой компоненты $E \subset X_{\bar{v}}$

$$
H^{0}\left(E, \Omega_{E}^{2}\right)=(0) .
$$

Если поверхность $V$ имеет стабильное сильное вырождение в некоторой точке $v$ поля $K$, то для любого конечного алгебраического расширения $K^{\prime}$ поля $K$ поверхность $V \otimes_{K} K^{\prime}$ имеет стабильное сильное вырождение в любой точке $v^{\prime}$ поля $K^{\prime}$, лежащей над $v[16$, предложение 1.3]. 
2.4. ТеОрема. Пусть $k(t)$ - поле рачиональных функиий алгебраически независимой переменной $t$ над числовым полем $k \hookrightarrow \mathbb{C},[k: \mathbb{Q}]<\infty, u$ пусть $V$ - гладкое проективное многообразие над $k(t)$. Предположим, что $H^{1}\left(V \otimes \overline{k(t)}, \mathscr{O}_{V \otimes \overline{k(t)}}\right)=(0), \quad \mathrm{NS}(V \otimes \overline{k(t)})=\mathrm{NS}(V), \quad V(k(t)) \neq \varnothing$, nростое число $l$ не делит $\operatorname{Card}\left([\mathrm{NS}(V \otimes \overline{k(t)})]_{\text {tors }}\right) u$

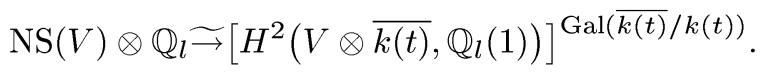

Тогда l-примарная компонента группы $\mathrm{Br}^{\prime}(V) / \operatorname{Br}(k(t))$ конечна.

ДоКАЗАТЕЛЬСТВо. Имеем

$$
\mathrm{NS}(V) \otimes \mathbb{Q}_{l} \underset{\rightarrow}{\widetilde{\sim}}\left[H^{2}\left(V \otimes \overline{k(t)}, \mathbb{Q}_{l}(1)\right)\right]^{\mathrm{Gal}(\overline{k(t)} / k(t))} .
$$

С другой стороны, $H^{1}\left(V \otimes \overline{k(t)}, \mathscr{O}_{V \otimes \overline{k(t)}}\right)=(0)$ - касательное пространство в нуле групшы $\operatorname{Pic}^{0}(V \otimes \overline{k(t)})\left[13\right.$, лекция 24]. Поэтому $\operatorname{Pic}^{0}(V \otimes \overline{k(t)})=(0)$. Следовательно,

$$
\operatorname{Pic}(V \otimes \overline{k(t)}) \simeq \operatorname{Pic}(V \otimes \overline{k(t)}) / \operatorname{Pic}^{0}(V \otimes \overline{k(t)})=\mathrm{NS}(V \otimes \overline{k(t)}),
$$

и точная последовательность (2.11) из [19] имеет вид

$$
\begin{aligned}
0 & \rightarrow \operatorname{Br}(k(t)) \rightarrow \operatorname{Ker}\left[\operatorname{Br}^{\prime}(V) \rightarrow \operatorname{Br}^{\prime}(V \otimes \overline{k(t)})^{\mathrm{Gal}(\overline{k(t)} / k(t))}\right] \\
& \rightarrow H^{1}(\operatorname{Gal}(\overline{k(t)} / k(t)), \mathrm{NS}(V \otimes \overline{k(t)})) \rightarrow 0 .
\end{aligned}
$$

Поскольку $\mathrm{NS}(V)=\mathrm{NS}(V \otimes \overline{k(t)})$, то группа $\mathrm{Gal}(\overline{k(t)} / k(t))$ действует тривиально на $\mathrm{NS}(V \otimes \overline{k(t)}) \simeq \mathbb{Z}^{\rho} \oplus[\mathrm{NS}(V \otimes \overline{k(t)})]_{\text {tors }}$ (неканонический изоморфизм, где $\rho=\operatorname{rank} \mathrm{NS}(V \otimes \overline{k(t)}))$. Следовательно,

$$
\begin{aligned}
& H^{1}(\operatorname{Gal}(\overline{k(t)} / k(t)), \operatorname{NS}(V \otimes \overline{k(t)}))=\operatorname{Hom}_{\text {cont }}(\operatorname{Gal}(\overline{k(t)} / k(t)), \operatorname{NS}(V \otimes \overline{k(t)})) \\
& \quad=\operatorname{Hom}_{\text {cont }}\left(\operatorname{Gal}(\overline{k(t)} / k(t)), \mathbb{Z}^{\rho}\right) \oplus \operatorname{Hom}_{\text {cont }}\left(\operatorname{Gal}(\overline{k(t)} / k(t)),[\operatorname{NS}(V \otimes \overline{k(t)})]_{\text {tors }}\right) \\
& \quad=\operatorname{Hom}_{\text {cont }}\left(\operatorname{Gal}(\overline{k(t)} / k(t)),[\operatorname{NS}(V \otimes \overline{k(t)})]_{\text {tors }}\right),
\end{aligned}
$$

так как $\mathbb{Z}^{\rho}$ не содержит нетривиальных компактных подгрупп, и в итоге мы имеем $\operatorname{Hom}_{\text {cont }}\left(\operatorname{Gal}(\overline{k(t)} / k(t)), \mathbb{Z}^{\rho}\right)=(0)$.

Поэтому точная последовательность (2.13) дает точную последовательность

$$
\begin{aligned}
0 & \rightarrow \operatorname{Br}(k(t)) \rightarrow \operatorname{Ker}\left[\operatorname{Br}^{\prime}(V) \rightarrow \operatorname{Br}^{\prime}(V \otimes \overline{k(t)}) \operatorname{Gal}(\overline{k(t)} / k(t))\right. \\
& \rightarrow H^{1}\left(\operatorname{Gal}(\overline{k(t)} / k(t)),[\mathrm{NS}(V \otimes \overline{k(t)})]_{\text {tors }}\right) \rightarrow 0 .
\end{aligned}
$$

Все группы в (2.14) являются группами кручения, и мы получаем точную последовательность $l$-примарных компонент

$$
\begin{aligned}
0 & \rightarrow \operatorname{Br}(k(t))(l) \rightarrow \operatorname{Ker}\left[\operatorname{Br}^{\prime}(V)(l) \rightarrow \operatorname{Br}^{\prime}(V \otimes \overline{k(t)})(l)^{\operatorname{Gal}(\overline{k(t)} / k(t))}\right] \\
& \rightarrow H^{1}\left(\operatorname{Gal}(\overline{k(t)} / k(t)),[\mathrm{NS}(V \otimes \overline{k(t)})]_{\mathrm{tors}}\right)(l) \rightarrow 0
\end{aligned}
$$


Заметим, что

$$
\begin{aligned}
& H^{1}\left(\operatorname{Gal}(\overline{k(t)} / k(t)),[\mathrm{NS}(V \otimes \overline{k(t)})]_{\text {tors }}\right)= \\
& \quad=\operatorname{Hom}_{\text {cont }}\left(\operatorname{Gal}(\overline{k(t)} / k(t)),[\operatorname{NS}(V \otimes \overline{k(t)})]_{\text {tors }}\right)
\end{aligned}
$$

аннулируется числом $\operatorname{Card}\left([\mathrm{NS}(V \otimes \overline{k(t)})]_{\mathrm{tors}}\right)$.

Если простое число $l$ не делит $\operatorname{Card}\left([\mathrm{NS}(V \otimes \overline{k(t)})]_{\mathrm{tors}}\right)$, то подгруппа $l$-кручения в $H^{1}\left(\mathrm{Gal}(\overline{k(t)} / k(t)),[\mathrm{NS}(V \otimes \overline{k(t)})]_{\text {tors }}\right)$ тривиальна, $(2.15)$ дает изоморфизм

$$
\operatorname{Br}(k(t))(l) \simeq \operatorname{Ker}\left[\operatorname{Br}^{\prime}(V)(l) \rightarrow \operatorname{Br}^{\prime}(V \otimes \overline{k(t)})(l)^{\operatorname{Gal}(\overline{k(t)} / k(t))}\right],
$$

и утверждение следует из конечности группы $\operatorname{Br}^{\prime}(V \otimes \overline{k(t)})(l)^{\mathrm{Gal}(\overline{k(t)} / k(t))}[19$, теорема 2.4]. Теорема доказана.

2.5. СлеДСТвИЕ. Пусть $k(t)$ - поле рациональных функций алгебраически независимой переменной $t$ над числовым полем $k \hookrightarrow \mathbb{C},[k: \mathbb{Q}]<\infty$, и пусть $V$ - гладкое проективное многообразие над $k(t)$. Предположим, что $H^{1}(V \otimes$ $\left.\overline{k(t)}, \mathscr{O}_{V \otimes \overline{k(t)}}\right)=(0), \mathrm{NS}(V \otimes \overline{k(t)})=\mathrm{NS}(V), \quad V(k(t)) \neq \varnothing$ и выполняется хотя бы одно из следующ,их условий:

a) $V$ - поверхность типа K3;

b) $V$ - поверхность со стабильным сильныцм вырождением в некоторой точке поля $k(t)$, и $l$ не делит $\operatorname{Card}\left([\mathrm{NS}(V \otimes \overline{k(t)})]_{\text {tors }}\right)$.

Тогда l-примарная компонента группы $\operatorname{Br}^{\prime}(V) / \operatorname{Br}(k(t))$ конечна.

ДоКАЗАТЕЛЬСТво. В силу теоремы 2.4 мы должны показать, что

$$
\mathrm{NS}(V) \otimes \mathbb{Q}_{l} \underset{\rightarrow}{\widetilde{T}}\left[H^{2}\left(V \otimes \overline{k(t)}, \mathbb{Q}_{l}(1)\right)\right]^{\mathrm{Gal}(\overline{k(t)} / k(t))} .
$$

В случае а) утверждение доказано в $[19, \S 3.3]$.

Пусть $V$ - поверхность со стабильным сильньм вырождением в некоторой точке $k(t)$ и $l$ не делит $\operatorname{Card}\left([\mathrm{NS}(V \otimes \overline{k(t)})]_{\text {tors }}\right)$. Тогда

$$
\mathrm{NS}(V \otimes \bar{k}(t)) \otimes \mathbb{Q}_{l} \underset{\rightarrow}{\widetilde{T}}\left[H^{2}\left(V \otimes \overline{k(t)}, \mathbb{Q}_{l}(1)\right)\right]^{\operatorname{Gal}(\overline{k(t)} / \bar{k}(t))}
$$

(см. [16, теорема 1.4] и принцип Лефшшеца, согласно которому можно рассматривать $\mathbb{C}$ вместо $\bar{k})$.

Легко видеть, что (2.16) дает следуюшее соотношение:

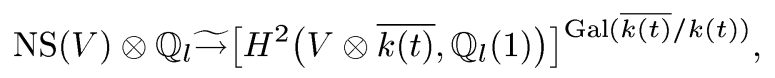

потому что

$$
\begin{gathered}
\mathrm{NS}(V \otimes \overline{k(t)}) \hookleftarrow \mathrm{NS}(V \otimes \bar{k}(t)) \hookleftarrow \mathrm{NS}(V), \\
\mathrm{NS}(V \otimes \overline{k(t)})=\mathrm{NS}(V), \\
\operatorname{Gal}(\overline{k(t)} / \bar{k}(t)) \hookrightarrow \operatorname{Gal}(\overline{k(t)} / k(t)) .
\end{gathered}
$$

Следствие доказано. 
2.6. В этом пункте мы доказываем теорему 0.4 .

Пусть $i_{y}: \operatorname{Spec}(k(y)) \rightarrow X$-каноническое вложение $y \in X$ и

$$
\operatorname{Div}_{X}^{\text {vert }}=\bigoplus_{\substack{y \in X \backslash V \\ \operatorname{codim}_{X}(y)=1}} i_{y^{*}} \mathbb{Z}
$$

- пучок вертикальных дивизоров Картье.

Существует точная последовательность пучков (в этальной топологии $X$ )

$$
0 \rightarrow \mathrm{G}_{m, X} \rightarrow h_{*} \mathrm{G}_{m, V} \rightarrow \operatorname{Div}_{X}^{\text {vert }} \rightarrow 0
$$

где $h: V \rightarrow X$ - вложение общего слоя $\pi$. Действительно, пусть $\eta$ - общая точка $\mathbb{P}_{k}^{1}$; тогда для любого этального морфизма $U \rightarrow X$ мы имеем коммутативную диаграмму

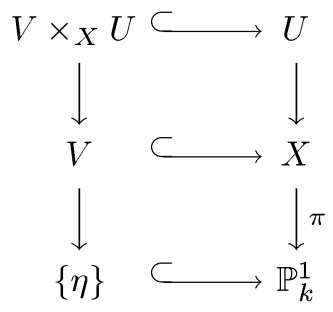

где каждый прямоугольник дает диаграмму расслоенного произведения. Мы видим, что $V \times_{X} U$ является общим слоем канонического морфизма $U \rightarrow \mathbb{P}_{k}^{1}$. Поскольку

$$
H^{0}\left(U, h_{*} \mathrm{G}_{m, V}\right)=H^{0}\left(V \times_{X} U, \mathrm{G}_{m}\right),
$$

то существует каноническое вложение $\mathrm{G}_{m, X} \rightarrow h_{*} \mathrm{G}_{m, V}$, которое на $U$ представляет собой вложение $H^{0}\left(U, \mathscr{O}_{U}^{\times}\right) \hookrightarrow H^{0}\left(V \times_{X} U, \mathscr{O}_{V \times_{X} U}^{\times}\right)$. Коядро этого отображения является пучком вертикальных дивизоров Картье $\mathrm{Div}_{X}^{\mathrm{vert}}$.

Имеем

$$
\operatorname{Div}_{X}=\bigoplus_{\substack{y \in X \\ \operatorname{codim}_{X}(y)=1}} i_{y^{*}} \mathbb{Z}=\left(\bigoplus_{\substack{y \in V \\ \operatorname{codim}_{X}(y)=1}} i_{y^{*}} \mathbb{Z}\right) \oplus \operatorname{Div}_{X}^{\text {vert }}
$$

С другой стороны, $H^{1}\left(X, \operatorname{Div}_{X}\right)=(0)[11$, гл. III, $\S 2$, пример 2.22]. Следовательно, $H^{1}\left(X, \operatorname{Div}_{X}^{\text {vert }}\right)=(0)$. Поэтому $(2.17)$ дает точную последовательность

$$
\begin{aligned}
0 & \rightarrow H^{2}\left(X, \mathrm{G}_{m}\right) \rightarrow H^{2}\left(X, h_{*} \mathrm{G}_{m, V}\right) \rightarrow H^{2}\left(X, \operatorname{Div}_{X}^{\text {vert }}\right) \\
\rightarrow H^{3}\left(X, \mathrm{G}_{m}\right) & \rightarrow H^{3}\left(X, h_{*} \mathrm{G}_{m, V}\right) \rightarrow H^{3}\left(X, \operatorname{Div}_{X}^{\text {vert }}\right)
\end{aligned}
$$

Спектральная последовательность Лере

$$
E_{2}^{p, q}=H^{p}\left(X, R^{q} h_{*} \mathrm{G}_{m, V}\right) \Longrightarrow H^{p+q}\left(V, \mathrm{G}_{m, V}\right)
$$

дает точную последовательность

$$
0 \rightarrow E_{2}^{1,0} \rightarrow E^{1} \rightarrow E_{2}^{0,1} \stackrel{d_{2}^{0,1}}{\rightarrow} E_{2}^{2,0} \rightarrow E_{1}^{2} \rightarrow E_{2}^{1,1} \rightarrow E_{2}^{3,0}
$$


где $E_{1}^{2}=\operatorname{Ker}\left[E^{2} \rightarrow E_{2}^{0,2}\right][11$, приложение В]. Поэтому мы имеем точную последовательность

$$
\begin{aligned}
0 & \rightarrow H^{1}\left(X, h_{*} \mathrm{G}_{m, V}\right) \rightarrow H^{1}\left(V, \mathrm{G}_{m}\right) \rightarrow H^{0}\left(X, R^{1} h_{*} \mathrm{G}_{m, V}\right) \stackrel{d_{2}^{0,1}}{\rightarrow} H^{2}\left(X, h_{*} \mathrm{G}_{m, V}\right) \\
& \rightarrow \operatorname{Ker}\left[H^{2}\left(V, \mathrm{G}_{m}\right) \rightarrow H^{0}\left(X, R^{2} h_{*} \mathrm{G}_{m, V}\right)\right] \rightarrow H^{1}\left(X, R^{1} h_{*} \mathrm{G}_{m, V}\right) .
\end{aligned}
$$

Слой $R^{1} h_{*} \mathrm{G}_{m, V}$ изоморфен $H^{1}\left(V \times_{X} \operatorname{Spec}\left(\mathscr{O}_{X, \bar{x}}\right), \mathrm{G}_{m}\right)$ в геометрической точке $\bar{x} \rightarrow x \in X[11$, гл. III, $\S 1$, теорема 1.15 , замечание $1.17(\mathrm{a})]$, где $\mathscr{O}_{X, \bar{x}}=\mathscr{O}_{X, x}^{\mathrm{sh}}-$ строгая гензелизация локального кольца $\mathscr{O}_{X, x}[11$, гл. I, $\S 4$, замечание 4.11]. Очевидно, что $\operatorname{Spec}\left(\mathscr{O}_{X, \bar{x}}\right)=\operatorname{Spec}\left(\mathscr{O}_{X, x}^{\mathrm{sh}}\right)$ является схемой над $\mathscr{O}_{\mathbb{P}_{k}^{1}, \pi(x)}$.

Напомним, что строгая гензелизация $\mathscr{O}_{X, x}^{\mathrm{sh}}$ регулярного локального нётерова кольца $\mathscr{O}_{X, x}$ является регулярным локальным нётеровым кольцом $[7$, предложение 18.8.8, следствие 18.8.13]. Следовательно, это кольцо факториально, и поэтомy $H^{1}\left(\operatorname{Spec}\left(\mathscr{O}_{X, x}^{\mathrm{sh}}\right), \mathrm{G}_{m}\right)=\operatorname{Pic}\left(\mathscr{O}_{X, x}^{\mathrm{sh}}\right)=(0)[8$, гл. II, $\S 6$, предложение 6.2, следствие 6.16].

Если $\bar{x} \rightarrow x \in V$, то $\pi(x)$ - обшая точка $\mathbb{P}_{k}^{1}$, поэтому $\operatorname{Spec}\left(\mathscr{O}_{X, \bar{x}}\right)$ - схема над $k(t)$, и $V \times_{X} \operatorname{Spec}\left(\mathscr{O}_{X, \bar{x}}\right)=\operatorname{Spec}\left(\mathscr{O}_{X, \bar{x}}\right)$. Следовательно,

$$
\begin{aligned}
\left(R^{1} h_{*} \mathrm{G}_{m, V}\right)_{\bar{x}} & =H^{1}\left(V \times_{X} \operatorname{Spec}\left(\mathscr{O}_{X, \bar{x}}\right), \mathrm{G}_{m}\right) \\
& =H^{1}\left(\operatorname{Spec}\left(\mathscr{O}_{X, \bar{x}}\right), \mathrm{G}_{m}\right)=\operatorname{Pic}\left(\operatorname{Spec}\left(\mathscr{O}_{X, \bar{x}}\right)\right)=(0) .
\end{aligned}
$$

Если $\bar{x} \rightarrow x \in X \backslash V$, то $\operatorname{Spec}\left(\mathscr{O}_{X, \bar{x}}\right)=\operatorname{Spec}\left(\mathscr{O}_{X, x}^{\mathrm{sh}}\right)$ - схема над дискретно нормированным кольцом $\mathscr{O}_{\mathbb{P}^{1}}, \pi(x)$. Пусть $\eta$ - обшая точка $\mathbb{P}_{k}^{1}$. Тогда мы имеем коммутативную диаграмму

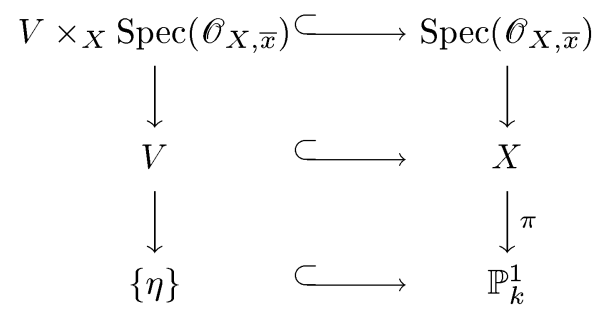

где каждый прямоугольник дает диаграмму расслоенного произведения. Видно, что $V \times_{X} \operatorname{Spec}\left(\mathscr{O}_{X}, \bar{x}\right)$ - обший слой канонического морфизма $\operatorname{Spec}\left(\mathscr{O}_{X}, \bar{x}\right) \rightarrow \mathbb{P}_{k}^{1}$. C другой стороны, морфизм $\operatorname{Spec}\left(\mathscr{O}_{X, \bar{x}}\right) \rightarrow \mathbb{P}_{k}^{1}$ является композицией канонических морфизмов

$$
\operatorname{Spec}\left(\mathscr{O}_{X, \bar{x}}\right) \rightarrow \operatorname{Spec}\left(\mathscr{O}_{X, x}\right) \rightarrow \operatorname{Spec}\left(\mathscr{O}_{\mathbb{P}_{k}^{1}, \pi(x)}\right) \hookrightarrow \mathbb{P}_{k}^{1} .
$$

Напомним, что $\mathscr{O}_{\mathbb{P}_{k}^{1}}, \pi(x)$ - дискретно нормированное кольцо. Следовательно, $V \times_{X} \operatorname{Spec}\left(\mathscr{O}_{X, \bar{x}}\right)-$ открытая подсхема в $\operatorname{Spec}\left(\mathscr{O}_{X, \bar{x}}\right)$. Поскольку $\operatorname{Spec}\left(\mathscr{O}_{X, \bar{x}}\right)$ и $V \times_{X} \operatorname{Spec}\left(\mathscr{O}_{X, \bar{x}}\right)$ - нётеровы регулярные схемы, то они локально факториальны. Поэтому сушествуют естественные изоморфизмы

$$
\begin{aligned}
\operatorname{Cl}\left(\operatorname{Spec}\left(\mathscr{O}_{X}, \bar{x}\right)\right) & \simeq \operatorname{Pic}\left(\operatorname{Spec}\left(\mathscr{O}_{X, \bar{x}}\right)\right), \\
\operatorname{Cl}\left(V \times_{X} \operatorname{Spec}\left(\mathscr{O}_{X, \bar{x}}\right)\right) & \simeq \operatorname{Pic}\left(V \times_{X} \operatorname{Spec}\left(\mathscr{O}_{X, \bar{x}}\right)\right)
\end{aligned}
$$


[8, гл. II, $\S 6$, следствие 6.16]. Поскольку $V \times_{X} \operatorname{Spec}\left(\mathscr{O}_{X, \bar{x}}\right)$ - открытая подсхема нётеровой регулярной схемы $\operatorname{Spec}\left(\mathscr{O}_{X, \bar{x}}\right)$, то существует точная последовательность

$$
\mathrm{Cl}\left(\operatorname{Spec}\left(\mathscr{O}_{X, \bar{x}}\right)\right) \rightarrow \mathrm{Cl}\left(V \times_{X} \operatorname{Spec}\left(\mathscr{O}_{X, \bar{x}}\right)\right) \rightarrow 0
$$

$\left[8\right.$, гл. II, $\S 6$, предложение 6.5]. Поэтому равенство $\operatorname{Pic}\left(\operatorname{Spec}\left(\mathscr{O}_{X}, \bar{x}\right)\right)=(0)$ дает равенство $\operatorname{Pic}\left(V \times_{X} \operatorname{Spec}\left(\mathscr{O}_{X}, \bar{x}\right)\right)=(0)$. В частности,

$$
\left(R^{1} h_{*} \mathrm{G}_{m, V}\right)_{\bar{x}}=H^{1}\left(V \times_{X} \operatorname{Spec}\left(\mathscr{O}_{X, \bar{x}}\right), \mathrm{G}_{m}\right)=(0) .
$$

Следовательно, $\left(R^{1} h_{*} \mathrm{G}_{m, V}\right)_{\bar{x}}=(0)$ для всех геометрических точек $\bar{x} \rightarrow x \in X$. Значит, $R^{1} h_{*} \mathrm{G}_{m, V}=(0)$, и (2.19) дает следуюший изоморфизм:

$$
H^{2}\left(X, h_{*} \mathrm{G}_{m, V}\right) \simeq \operatorname{Ker}\left[\operatorname{Br}^{\prime}(V) \rightarrow H^{0}\left(X, R^{2} h_{*} \mathrm{G}_{m, V}\right)\right] .
$$

Поэтому (2.18) дает точную последовательность

$$
0 \rightarrow \operatorname{Br}^{\prime}(X) \rightarrow \operatorname{Ker}\left[\operatorname{Br}^{\prime}(V) \rightarrow H^{0}\left(X, R^{2} h_{*} \mathrm{G}_{m, V}\right)\right] \rightarrow H^{2}\left(X, \operatorname{Div}_{X}^{\text {vert }}\right) .
$$

Начиная с этого места мы обозначаем через $k(y)^{s}$ сепарабельное замыкание $k(y)$ в $\overline{k(y)}$. Поскольку $V(k(t)) \neq \varnothing$, то сушествует каноническое вложение $\operatorname{Br}(k(t)) \hookrightarrow$ $\operatorname{Br}^{\prime}(V)$.

С другой стороны, сушествует каноническая точная последовательность

$$
\begin{aligned}
0 & \rightarrow \operatorname{Br}\left(\mathbb{P}_{k}^{1}\right) \rightarrow \operatorname{Br}(k(t)) \rightarrow \bigoplus_{\operatorname{codim}_{\mathbb{P}_{k}^{1}}(v)=1} \operatorname{Hom}_{\text {cont }}\left(\operatorname{Gal}\left(k(v)^{s} / k(v)\right), \mathbb{Q} / \mathbb{Z}\right) \\
& \rightarrow H^{3}\left(\mathbb{P}_{k}^{1}, \mathrm{G}_{m}\right) \rightarrow H^{3}\left(\operatorname{Spec}(k(t)), \mathrm{G}_{m}\right)
\end{aligned}
$$

[11, гл. III, $\S 3$, пример 2.22(a)].

Пусть $g: \operatorname{Spec}(k(t)) \hookrightarrow \mathbb{P}_{k}^{1}$ - вложение общей точки $\mathbb{P}_{k}^{1}$. Мы имеем точную последовательность пучков в этальной топологии $\mathbb{P}_{k}^{1}$ :

$$
0 \rightarrow \mathrm{G}_{m, \mathbb{P}_{k}^{1}} \rightarrow g_{*} \mathrm{G}_{m, k(t)} \rightarrow \operatorname{Div}_{\mathbb{P}_{k}^{1}} \rightarrow 0
$$

[11, гл. III, $\S 2$, пример 2.22]. Рассмотрим спектральную последовательность Лере

$$
H^{p}\left(\mathbb{P}_{k}^{1}, R^{q} g_{*} \mathrm{G}_{m, k(t)}\right) \Longrightarrow H^{p+q}\left(\operatorname{Spec}(k(t)), \mathrm{G}_{m}\right) .
$$

Поскольку $\mathbb{P}_{k}^{1}$ - превосходная схема, мы имеем $R^{q} g_{*} \mathrm{G}_{m, k(t)}=(0)$ для $q>0[11$, гл. III, $\S 2$, пример $2.22(\mathrm{~b})]$. Поэтому точная последовательность

$$
0 \rightarrow E_{2}^{1,0} \rightarrow E^{1} \rightarrow E_{2}^{0,1} \stackrel{d_{2}^{0,1}}{\rightarrow} E_{2}^{2,0} \rightarrow E_{1}^{2} \rightarrow E_{2}^{1,1}
$$

$\left(\right.$ где $\left.E_{1}^{2}=\operatorname{Ker}\left[E^{2} \rightarrow E_{2}^{0,2}\right]\right)[11$, приложение В] дает соотношение

$$
H^{2}\left(\operatorname{Spec}(k(t)), \mathrm{G}_{m}\right)=H^{2}\left(\mathbb{P}_{k}^{1}, g_{*} \mathrm{G}_{m, k(t)}\right) ;
$$

так как $H^{1}\left(\mathbb{P}_{k}^{1}, \operatorname{Div}_{\mathbb{P}_{k}^{1}}\right)=(0)[11$, гл. III, $\S 2$, пример 2.22], то мы получаем из $(2.21)$ точную последовательность

$$
0 \rightarrow H^{2}\left(\mathbb{P}_{k}^{1}, \mathrm{G}_{m}\right) \rightarrow H^{2}\left(\operatorname{Spec}(k(t)), \mathrm{G}_{m}\right) \rightarrow H^{2}\left(\mathbb{P}_{k}^{1}, \operatorname{Div}_{\mathbb{P}_{k}^{1}}\right)
$$


Соотношение $V(k(t)) \neq \varnothing$ показывает, что сушествует сечение $\theta: \mathbb{P}_{k}^{1} \rightarrow X$. Легко видеть, что для любой геометрической точки $\bar{v} \rightarrow v$ индекс пересечения $\left(\theta\left(\mathbb{P}_{\bar{k}}\right) \cdot X \otimes \bar{k} \pi^{-1}(\bar{v})\right)$ равен 1 . Следовательно, $\theta\left(\mathbb{P}_{k}^{1}\right)$ пересекается в точности с одной неприводимой компонентой любого схемного слоя $\pi^{-1}(v), v \in \mathbb{P}_{k}^{1}$.

Поэтому любой вертикальный дивизор Картье $D$ с носителем в $\pi^{-1}(v)$ может быть единственньмм образом представлен в виде

$$
D=n_{0} \cdot \pi^{-1}(v)+\sum_{i} n_{i} \cdot D_{i}
$$

где $n_{j} \in \mathbb{Z}, \quad j=0,1, \ldots, \quad D_{i}$ - такие неприводимые компоненты $\pi^{-1}(v)$, что $D_{i} \cap \theta\left(\mathbb{P}_{k}^{1}\right)=\varnothing$, и мы получаем следующее разложение пучков:

$$
\operatorname{Div}_{X}^{\text {vert }}=\pi^{*}\left(\operatorname{Div}_{\mathbb{P}_{k}^{1}}\right) \oplus\left(\bigoplus_{\substack{y \in X \backslash V \\ \operatorname{codim}_{X}(y)=1 \\\{y\} \cap \theta\left(\mathbb{P}_{k}^{1}\right)=\varnothing}} i_{y^{*}} \mathbb{Z}\right) .
$$

Это разложение определяет инъективные морфизмы

$$
\pi^{*}: \operatorname{Br}(k(t)) \hookrightarrow \operatorname{Br}^{\prime}(V), \quad H^{2}\left(\mathbb{P}_{k}^{1}, \operatorname{Div}_{\mathbb{P}_{k}^{1}}\right) \hookrightarrow H^{2}\left(X, \operatorname{Div}_{X}^{\text {vert }}\right)
$$

(см. $[19, \S 6.1])$.

Пусть $B=\operatorname{Ker}\left[\operatorname{Br}^{\prime}(V) \rightarrow H^{0}\left(X, R^{2} h_{*} \mathrm{G}_{m, V}\right)\right]$. Заметим, что $H^{2}\left(\mathbb{P}_{k}^{1}, \mathrm{G}_{m}\right)=$ $\operatorname{Br}\left(\mathbb{P}_{k}^{1}\right)=\operatorname{Br}(k)[19$, лемма 2.1]. С другой стороны, $(2.20)$ и (2.22) дают коммутативную диаграмму с точными строками

$$
\begin{aligned}
& 0 \longrightarrow H^{2}\left(\mathbb{P}_{k}^{1}, \mathrm{G}_{m}\right) \longrightarrow H^{2}\left(\operatorname{Spec}(k(t)), \mathrm{G}_{m}\right) \cap B \longrightarrow H^{2}\left(\mathbb{P}_{k}^{1}, \operatorname{Div}_{\mathbb{P}_{k}^{1}}\right) \\
& \cap \cap \cap \cap \cap \\
& 0 \longrightarrow \operatorname{Br}^{\prime}(X) \longrightarrow B \quad \longrightarrow H^{2}\left(X, \operatorname{Div}_{X}^{\text {vert }}\right)
\end{aligned}
$$

Пусть $C=\operatorname{Im}\left[H^{2}\left(\operatorname{Spec}(k(t)), \mathrm{G}_{m}\right) \cap B \rightarrow H^{2}\left(\mathbb{P}_{k}^{1}, \operatorname{Div}_{\mathbb{P}_{k}^{1}}\right)\right]$. Тогда (2.23) дает коммутативную диаграмму с точными строками

$$
\begin{array}{ccc}
0 \longrightarrow \operatorname{Br}(k) & \longrightarrow \operatorname{Br}(k(t)) \cap B \longrightarrow 0 \\
\cap & \cap & C \\
0 \longrightarrow \operatorname{Br}^{\prime}(X) \longrightarrow & B & \longrightarrow
\end{array}
$$

Мы получаем змеевидную диаграмму

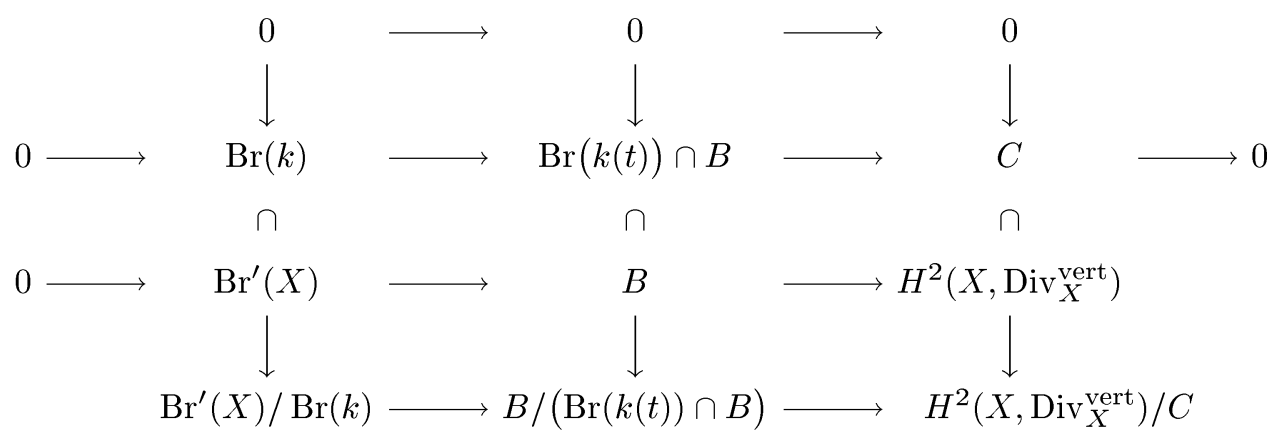


с точной последовательностью

$$
0 \rightarrow \mathrm{Br}^{\prime}(X) / \operatorname{Br}(k) \rightarrow B /(\operatorname{Br}(k(t)) \cap B) \rightarrow H^{2}\left(X, \operatorname{Div}_{X}^{\text {vert }}\right) / C
$$

$[2, \S 1$, предложение 2]. С другой стороны, $B /(\operatorname{Br}(k(t)) \cap B)$ является образом подгруппы $B \hookrightarrow \mathrm{Br}^{\prime}(V)$ в $\mathrm{Br}^{\prime}(V) / \mathrm{Br}(k(t))$ при каноническом морфизме $\mathrm{Br}^{\prime}(V) \rightarrow$ $\operatorname{Br}^{\prime}(V) / \operatorname{Br}(k(t))$. Поэтому мы имеем точную последовательность

$$
0 \rightarrow \mathrm{Br}^{\prime}(X) / \mathrm{Br}(k) \rightarrow \mathrm{Br}^{\prime}(V) / \mathrm{Br}(k(t)) .
$$

Предположим, что $H^{1}\left(V \otimes \overline{k(t)}, \mathscr{O}_{V \otimes \overline{k(t)}}\right)=(0), \mathrm{NS}(V \otimes \overline{k(t)})=\mathrm{NS}(V)$, простое число $l$ не делит $\operatorname{Card}\left([\mathrm{NS}(V \otimes \overline{k(t)})]_{\text {tors }}\right)$ и

$$
\mathrm{NS}(V) \otimes \mathbb{Q}_{l} \underset{\rightarrow}{\sim}\left[H^{2}\left(V \otimes \overline{k(t)}, \mathbb{Q}_{l}(1)\right)\right]^{\mathrm{Gal}(\overline{k(t)} / k(t))} .
$$

В силу теоремы $2.4 l$-компонента группы $\mathrm{Br}^{\prime}(V) / \mathrm{Br}(k(t))$ конечна. Следовательно, (2.24) дает конечность $l$-компоненты группы $\operatorname{Br}^{\prime}(X) / \operatorname{Br}(k)$.

Остается показать конечность $l$-компоненты группы $\mathrm{Br}^{\prime}(\Xi)$.

Пусть $i_{y}: \operatorname{Spec}(k(y)) \rightarrow \Xi-$ каноническое вложение $y \in \Xi$ и

$$
\operatorname{Div} \text { vert }^{\text {vert }}=\bigoplus_{\substack{y \in \Xi \backslash X \\ \operatorname{codim}_{\Xi}(y)=1}} i_{y * \mathbb{Z}}
$$

- пучок вертикальных дивизоров Картье.

Сушествует точная последовательность пучков (в этальной топологии $\Xi$ )

$$
0 \rightarrow \mathrm{G}_{m, \Xi} \rightarrow \nu_{*} \mathrm{G}_{m, X} \rightarrow \operatorname{Div}_{\Xi}^{\text {vert }} \rightarrow 0
$$

где $\nu: X \rightarrow \Xi-$ вложение обшего слоя $\varphi[19,(6.1)]$. Она дает точную последовательность

$$
0 \rightarrow \operatorname{Br}^{\prime}(\Xi) \rightarrow \operatorname{Ker}\left[\operatorname{Br}^{\prime}(X) \rightarrow H^{0}\left(\Xi, R^{2} \nu_{*} \mathrm{G}_{m, X}\right)\right] \rightarrow H^{2}\left(\Xi, \operatorname{Div}_{\Xi}^{\text {vert }}\right)
$$

(см. $[19,(6.4)])$.

Напомним, что (2.8) имеет вид

$$
0 \rightarrow \operatorname{Br}(k)^{\text {неapx }} \rightarrow H^{2}\left(\operatorname{Spec}(A), \operatorname{Div}_{\operatorname{Spec}(A)}\right)
$$

где $\operatorname{Br}(k)^{\text {неарх }} \hookrightarrow \operatorname{Br}(k)$ - подгруппа конечного индекса.

Пусть $\theta: \operatorname{Spec}(A) \rightarrow \Xi-$ сечение морфизма $\varphi$, удовлетворяющее условиям теоремы. Оно определяет инъективные морфизмы

$$
\operatorname{Br}(k) \hookrightarrow \operatorname{Br}^{\prime}(X), \quad H^{2}\left(\operatorname{Spec}(A), \operatorname{Div}_{\operatorname{Spec}(A)}\right) \hookrightarrow H^{2}\left(\Xi, \operatorname{Div}_{\Xi}^{\text {vert }}\right)
$$

$[19,(6.12)]$. Пусть $B^{\prime}=\operatorname{Ker}\left[\operatorname{Br}^{\prime}(X) \rightarrow H^{0}\left(\Xi, R^{2} \nu_{*} \mathrm{G}_{m, X}\right)\right]$. Очевидно, что (2.25) и (2.26) дают коммутативную диаграмму с точными строками

$$
\begin{aligned}
0 \longrightarrow \operatorname{Br}^{\prime}(\Xi) \longrightarrow & B^{\prime} \quad \longrightarrow \quad H^{2}\left(\Xi, \operatorname{Div}_{\Xi}^{\text {vert }}\right) \\
\cup & \longrightarrow \\
0 & \operatorname{Br}(k)^{\text {Heapx }} \cap B^{\prime} \longrightarrow H^{2}\left(\operatorname{Spec}(A), \operatorname{Div}_{\operatorname{Spec}(A)}\right) .
\end{aligned}
$$

Значит, $\operatorname{Br}^{\prime}(\Xi) \cap \operatorname{Br}(k)^{\text {неарх }}=(0)$ внутри $\operatorname{Br}^{\prime}(X)$. Поэтому

$$
\operatorname{Br}^{\prime}(\Xi) \hookrightarrow \operatorname{Br}^{\prime}(X) / \operatorname{Br}(k)^{\text {неарх }}
$$

с другой стороны, существует точная последовательность

$$
0 \rightarrow \operatorname{Br}(k) / \operatorname{Br}(k)^{\text {неарх }} \rightarrow \mathrm{Br}^{\prime}(X) / \operatorname{Br}(k)^{\text {неарх }} \rightarrow \mathrm{Br}^{\prime}(X) / \operatorname{Br}(k) \rightarrow 0
$$

с конечной группой $\operatorname{Br}(k) / \operatorname{Br}(k)^{\text {неарх }}$. Поскольку $l$-примарная компонента группы $\operatorname{Br}^{\prime}(X) / \operatorname{Br}(k)$ конечна, мы получаем конечность $l$-компоненты групшы $\mathrm{Br}^{\prime}(\Xi)$. Теорема 0.4 доказана. 
2.7. СлЕДСтвИЕ. Пусть $k(t)$ - поле рачиональных функиий алгебраически независимой переменной $t$ над числовым полем $k \hookrightarrow \mathbb{C},[k: \mathbb{Q}]<\infty, u$ пусть $V$ - гладкое проективное многообразие над $k(t)$. Предположим, что $H^{1}\left(V \otimes \overline{k(t)}, \mathscr{O}_{V \otimes \overline{k(t)}}\right)=(0), \quad \mathrm{NS}(V \otimes \overline{k(t)})=\mathrm{NS}(V), \quad V(k(t)) \neq \varnothing$ и выполняется хотя бы одно из следующих условий:

a) $V$ - поверхность типа $\mathrm{K} 3$;

b) $V$ - поверхность со стабильным сильныцм вырождением в некоторой точке поля $k(t)$ u $l$ не делит $\operatorname{Card}\left([\mathrm{NS}(V \otimes \overline{k(t)})]_{\text {tors }}\right)$.

Пусть $\pi: X \rightarrow \mathbb{P}_{k}^{1}-$ собственный плоский $k$-морфизм гладких проективных многообразий над $k$, и общий слой $\pi$ изоморфен $V$ (мь называем $\pi: X \rightarrow \mathbb{P}_{k}^{1}$ геометрической моделью $V$ ).

Тогда существует естественное вложение

$$
\operatorname{Br}^{\prime}(X) / \operatorname{Br}(k) \hookrightarrow \operatorname{Br}^{\prime}(V) / \operatorname{Br}(k(t))
$$

u l-примарная компонента группы $\operatorname{Br}^{\prime}(X) / \operatorname{Br}(k)$ конечна.

Это вытекает непосредственно из следствия 2.5 и теоремы 0.4 .

2.8. В этом пункте мы доказываем теорему 0.5 .

Согласно теореме $0.4 l$-примарная компонента группы $\mathrm{Br}^{\prime}(X) / \mathrm{Br}(k)$ конечна; поэтому $H^{1}\left(X \otimes \bar{k}, \mathscr{O}_{X \otimes \bar{k}}\right)=(0)[19$, теорема 5.3] и точная последовательность (2.1) имеет вид

$$
\begin{aligned}
0 & \rightarrow \operatorname{Br}(k) \rightarrow \operatorname{Ker}\left[\operatorname{Br}^{\prime}(X) \rightarrow \operatorname{Br}^{\prime}(X \otimes \bar{k})^{\operatorname{Gal}(\bar{k} / k)}\right] \\
& \rightarrow H^{1}(\operatorname{Gal}(\bar{k} / k), \operatorname{NS}(X \otimes \bar{k})) \rightarrow 0 .
\end{aligned}
$$

Поскольку $\mathrm{NS}(X \otimes \bar{k})=\mathrm{NS}(X)$, то группа Галуа $\mathrm{Gal}(\bar{k} / k)$ действует тривиально на $\mathrm{NS}(X \otimes \bar{k})$. Поэтому в рассматриваемом случае мы получаем точную последовательность (2.3) $l$-примарных компонент:

$$
\begin{aligned}
0 & \rightarrow \operatorname{Br}(k)(l) \rightarrow \operatorname{Ker}\left[\operatorname{Br}^{\prime}(X)(l) \rightarrow \operatorname{Br}^{\prime}(X \otimes \bar{k})(l)^{\operatorname{Gal}(\bar{k} / k)}\right] \\
& \rightarrow H^{1}\left(\operatorname{Gal}(\bar{k} / k),[\mathrm{NS}(X \otimes \bar{k})]_{\text {tors }}\right)(l) \rightarrow 0 .
\end{aligned}
$$

Заметим, что $H^{1}\left(\operatorname{Gal}(\bar{k} / k),[\mathrm{NS}(X \otimes \bar{k})]_{\text {tors }}\right)=\operatorname{Hom}_{\text {cont }}\left(\operatorname{Gal}(\bar{k} / k),[\mathrm{NS}(X \otimes \bar{k})]_{\text {tors }}\right)$ аннулируется числом $\operatorname{Card}\left([\mathrm{NS}(X \otimes \bar{k})]_{\text {tors }}\right)$. Подгруппа $l$-кручения в $H^{1}(\mathrm{Gal}(\bar{k} / k)$, $\left.[\mathrm{NS}(X \otimes \bar{k})]_{\text {tors }}\right)$ тривиальна, так как $l$ не делит $\operatorname{Card}\left([\mathrm{NS}(X \otimes \bar{k})]_{\text {tors }}\right)$. Следовательно, (2.27) дает изоморфизм

$$
\operatorname{Br}(k)(l) \simeq \operatorname{Ker}\left[\operatorname{Br}^{\prime}(X)(l) \rightarrow \operatorname{Br}^{\prime}(X \otimes \bar{k})(l)^{\operatorname{Gal}(\bar{k} / k)}\right] .
$$

С другой стороны, сюръективность канонического морфизма

$$
\operatorname{Br}^{\prime}(X)(l) \rightarrow \operatorname{Br}^{\prime}(X \otimes \bar{k})(l)^{\operatorname{Gal}(\bar{k} / k)}
$$

дает конечность групшы $\mathrm{Br}^{\prime}(X \otimes \bar{k})(l)^{\mathrm{Gal}(\bar{k} / k)}$, так как группа

$$
\operatorname{Im}\left[\operatorname{Br}^{\prime}(X)(l) \rightarrow \operatorname{Br}^{\prime}(X \otimes \bar{k})(l)^{\operatorname{Gal}(\bar{k} / k)}\right] \simeq \operatorname{Br}^{\prime}(X)(l) / \operatorname{Br}(k)(l)
$$

конечна. 
Хорошо известно, что $l$-примарная компонента когомологической групшы Брауэра $\mathrm{Br}^{\prime}(X \otimes \bar{k})=H^{2}\left(X \otimes \bar{k}, \mathrm{G}_{m}\right)$ изоморфна $\left(\mathbb{Q}_{l} / \mathbb{Z}_{l}\right)^{\rho_{0}(X \otimes \bar{k}, l)} \oplus$ (конечная группа) $[11$, гл. V,$\S 3$, замечание $3.29(\mathrm{~d})]$. Следовательно,

$$
T_{l} \operatorname{Br}^{\prime}(X \otimes \bar{k}) \stackrel{\text { def }}{=} \lim _{\longleftarrow} \operatorname{Br}^{\prime}(X \otimes \bar{k})_{l^{m}}=\operatorname{Hom}\left(\mathbb{Q}_{l} / \mathbb{Z}_{l}, \operatorname{Br}^{\prime}(X \otimes \bar{k})\right)
$$

- свободный конечно порожденный $\mathbb{Z}_{l}$-модуль ранга $\rho_{0}(X \otimes \bar{k}, l)$. Последовательность Куммера

$$
1 \rightarrow \mu_{l^{m}} \rightarrow \mathrm{G}_{m} \stackrel{l^{m}}{\rightarrow} \mathrm{G}_{m} \rightarrow 1
$$

дает точную последовательность

$$
\begin{aligned}
\cdots & \rightarrow H^{1}\left(X \otimes \bar{k}, \mathrm{G}_{m}\right) \stackrel{l^{m}}{\rightarrow} H^{1}\left(X \otimes \bar{k}, \mathrm{G}_{m}\right) \rightarrow H^{2}\left(X \otimes \bar{k}, \mu_{l^{m}}\right) \\
& \rightarrow H^{2}\left(X \otimes \bar{k}, \mathrm{G}_{m}\right) \stackrel{l^{m}}{\rightarrow} H^{2}\left(X \otimes \bar{k}, \mathrm{G}_{m}\right) \rightarrow \cdots
\end{aligned}
$$

Следовательно, мы имеем точную последовательность $\mathrm{Gal}(\bar{k} / k)$-модулей

$$
0 \rightarrow \mathrm{NS}(X \otimes \bar{k}) \otimes \mathbb{Z}_{l} \rightarrow H^{2}\left(X \otimes \bar{k}, \mathbb{Z}_{l}(1)\right) \rightarrow T_{l} \operatorname{Br}^{\prime}(X \otimes \bar{k}) \rightarrow 0
$$

$[11$, гл. V,$\S 3$, замечание $3.29(\mathrm{~d})]$.

Точная последовательность (2.28) дает точную последовательность

$$
\begin{aligned}
0 & \rightarrow\left[\mathrm{NS}(X \otimes \bar{k}) \otimes \mathbb{Q}_{l}\right]^{\operatorname{Gal}(\bar{k} / k)} \rightarrow\left[H^{2}\left(X \otimes \bar{k}, \mathbb{Q}_{l}(1)\right)\right]^{\operatorname{Gal}(\bar{k} / k)} \\
& \rightarrow\left[\left[T_{l} \operatorname{Br}^{\prime}(X \otimes \bar{k})\right] \otimes \mathbb{Q}_{l}\right]^{\operatorname{Gal}(\bar{k} / k)} \\
& \rightarrow H^{1}\left(\operatorname{Gal}(\bar{k} / k), \mathrm{NS}(X \otimes \bar{k}) \otimes \mathbb{Q}_{l}\right) \rightarrow \cdots
\end{aligned}
$$

Для любой открытой нормальной подгрупшы $U \mathbb{Q}_{l}$-пространство

$$
H^{1}\left(\mathrm{Gal}(\bar{k} / k) / U,\left[\mathrm{NS}(X \otimes \bar{k}) \otimes \mathbb{Q}_{l}\right]^{U}\right)=H^{1}\left(\operatorname{Gal}(\bar{k} / k) / U,\left[\mathrm{NS}(X) \otimes \mathbb{Q}_{l}\right]^{U}\right)
$$

аннулируется числом $\operatorname{Card}(\operatorname{Gal}(\bar{k} / k) / U)[4$, гл. IV,$\S 6$, следствие 1]. Значит,

$$
\begin{gathered}
H^{1}\left(\operatorname{Gal}(\bar{k} / k) / U,\left[\operatorname{NS}(X \otimes \bar{k}) \otimes \mathbb{Q}_{l}\right]^{U}\right)=(0), \\
H^{1}\left(\operatorname{Gal}(\bar{k} / k), \mathrm{NS}(X \otimes \bar{k}) \otimes \mathbb{Q}_{l}\right)=\lim _{\longrightarrow} H^{1}\left(\operatorname{Gal}(\bar{k} / k) / U,\left[\operatorname{NS}(X \otimes \bar{k}) \otimes \mathbb{Q}_{l}\right]^{U}\right)=(0) .
\end{gathered}
$$

Поэтому (2.29) дает точную последовательность

$$
\begin{aligned}
0 & \rightarrow \mathrm{NS}(X) \otimes \mathbb{Q}_{l} \rightarrow\left[H^{2}\left(X \otimes \bar{k}, \mathbb{Q}_{l}(1)\right)\right]^{\operatorname{Gal}(\bar{k} / k)} \\
& \rightarrow\left[\left[T_{l} \operatorname{Br}^{\prime}(X \otimes \bar{k})\right] \otimes \mathbb{Q}_{l}\right]^{\operatorname{Gal}(\bar{k} / k)} \rightarrow 0 .
\end{aligned}
$$

Так как $T_{l} \operatorname{Br}^{\prime}(X \otimes \bar{k})$ - свободньй конечно порожденный $\mathbb{Z}_{l}$-модуль, то мы видим, что $\left[T_{l} \operatorname{Br}^{\prime}(X \otimes \bar{k})\right]^{\operatorname{Gal}(\bar{k} / k)}$ - свободный конечно порожденный $\mathbb{Z}_{l}$-модуль.

С другой стороны,

$$
\begin{aligned}
& {\left[T_{l} \operatorname{Br}^{\prime}(X \otimes \bar{k})\right]^{\operatorname{Gal}(\bar{k} / k)}=\left[\operatorname{Hom}\left(\mathbb{Q}_{l} / \mathbb{Z}_{l}, \operatorname{Br}^{\prime}(X \otimes \bar{k})\right)\right]^{\operatorname{Gal}(\bar{k} / k)}} \\
& \quad=\operatorname{Hom}\left(\mathbb{Q}_{l} / \mathbb{Z}_{l},\left[\operatorname{Br}^{\prime}(X \otimes \bar{k})\right]^{\operatorname{Gal}(\bar{k} / k)}(l)\right)=T_{l}\left[\operatorname{Br}^{\prime}(X \otimes \bar{k})^{\operatorname{Gal}(\bar{k} / k)}(l)\right] .
\end{aligned}
$$

Напомним, что $\operatorname{Br}^{\prime}(X \otimes \bar{k})^{\operatorname{Gal}(\bar{k} / k)}(l)$ - конечная группа. Поэтому

$$
T_{l}\left[\operatorname{Br}^{\prime}(X \otimes \bar{k})^{\operatorname{Gal}(\bar{k} / k)}(l)\right]=(0) .
$$

Следовательно, $\left[\left[T_{l} \operatorname{Br}^{\prime}(X \otimes \bar{k})\right] \otimes \mathbb{Q}_{l}\right]^{\mathrm{Gal}(\bar{k} / k)}=(0)$, и $(2.30)$ дает изоморфизм

$$
\mathrm{NS}(X) \otimes \mathbb{Q}_{l} \simeq\left[H^{2}\left(X \otimes \bar{k}, \mathbb{Q}_{l}(1)\right)\right]^{\mathrm{Gal}(\bar{k} / k)} .
$$

Теорема 0.5 доказана. 
2.9. СЛЕДСТВИЕ. Пусть $V$ - гладкое проективное многообразие над $k(t)$, где $k(t)$ - поле рачиональных функиий алгебраически независимой переменной $t$ над числовым полем $k \hookrightarrow \mathbb{C},[k: \mathbb{Q}]<\infty$, и пусть $\pi: X \rightarrow \mathbb{P}_{k}^{1}-$ такой собственный плоский $k$-морфизм гладких проективных $k$-многообразий, что общий слой $\pi$ изоморфен $V\left(\right.$ мь называем $\pi: X \rightarrow \mathbb{P}_{k}^{1}$ геометрической моделью $V)$. Предположим, что $H^{1}\left(V \otimes \overline{k(t)}, \mathscr{O}_{V \otimes \overline{k(t)}}\right)=(0), \quad \mathrm{NS}(V \otimes$ $\overline{k(t)})=\mathrm{NS}(V), \quad V(k(t)) \neq \varnothing, \quad \mathrm{NS}(X \otimes \bar{k})=\mathrm{NS}(X)$, простое число $l$ не делит $\operatorname{Card}\left([\mathrm{NS}(V \otimes \overline{k(t)})]_{\text {tors }}\right)$ u $\operatorname{Card}\left([\mathrm{NS}(X \otimes \bar{k})]_{\text {tors }}\right)$, канонический морфизм

$$
\operatorname{Br}^{\prime}(X)(l) \rightarrow\left[\mathrm{Br}^{\prime}(X \otimes \bar{k})(l)\right]^{\mathrm{Gal}(\bar{k} / k)}
$$

сюрвективен и выполняется хотя бы одно из следующих условий:

а) $V$ - поверхность типа $\mathrm{K} 3$;

b) $V$ - поверхность со стабильным сильным вырождением в некоторой точке поля $k(t)$.

Тогда существует канонический изоморфизм

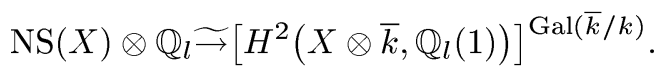

ДокАЗАТЕЛЬСтво. Это следует непосредственно из теоремы 0.5, потому что в п. 2.5 мы доказали соотношение

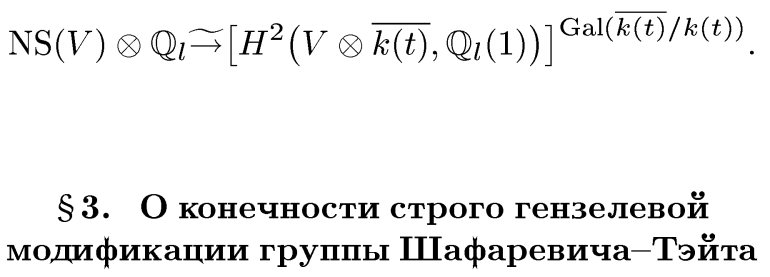

3.1. Для любого поля $L$ мы обозначаем через $\bar{L}$ максимальное сепарабельное алгебраическое расширение $L$.

Пусть $C$ - регулярная превосходная неприводимая 1 -мерная схема, $K$ - поле рациональных функций на $C$. Пусть $\widehat{K}_{v}$ - пополнение $K$ относительно нормирования, определенного замкнутой точкой $v \in C$. Множество всех таких пополнений используется для определения группы Шаффаревича-Тэйта абелева многообразия $J$ над $K$ :

$$
\mathrm{III}(C, J)=\operatorname{Ker}\left[H^{1}(\operatorname{Spec}(K), J) \rightarrow \prod_{\operatorname{codim}_{C}(v)=1} H^{1}\left(\operatorname{Spec}\left(\widehat{K}_{v}\right), J\right)\right]
$$

Имеет смысл использовать обычную гензелизацию и строгую гензелизацию локального кольца $\mathscr{O}_{C, v}$ вместо пополнения и определить гензелеву и строго гензелеву модификации группы Шафаревича-Тэйта. 
3.2. ОПРЕДЕЛЕниЕ. Пусть $K_{v}^{\mathrm{h}}\left(\right.$ соответственно $\left.K_{v}^{\mathrm{sh}}\right)$ - поле дробей обычной гензелизации $\mathscr{O}_{C, v}^{\mathrm{h}}$ (соответственно строгой гензелизации $\left.\mathscr{O}_{C, v}^{\mathrm{sh}}=\mathscr{O}_{C, \bar{v}}\right)$ локального кольца $\mathscr{O}_{C, v}$. Для абелева многообразия $J$ над $K$ определим

$$
\begin{gathered}
\mathrm{III}^{\mathrm{h}}(C, J)=\operatorname{Ker}\left[H^{1}(\operatorname{Spec}(K), J) \rightarrow \prod_{\operatorname{codim}_{C}(v)=1} H^{1}\left(\operatorname{Spec}\left(K_{v}^{\mathrm{h}}\right), J\right)\right], \\
\mathrm{III}^{\mathrm{sh}}(C, J)=\operatorname{Ker}\left[H^{1}(\operatorname{Spec}(K), J) \rightarrow \prod_{\operatorname{codim}_{C}(v)=1} H^{1}\left(\operatorname{Spec}\left(K_{v}^{\mathrm{sh}}\right), J\right)\right] .
\end{gathered}
$$

Очевидно, что вложения $K \hookrightarrow K_{v}^{\mathrm{h}} \hookrightarrow K_{v}^{\mathrm{sh}}$ и $K \hookrightarrow K_{v}^{\mathrm{h}} \hookrightarrow \widehat{K}_{v}$ индуцируют включения $\operatorname{III}^{\mathrm{h}}(C, J) \hookrightarrow \mathrm{III}^{\mathrm{sh}}(C, J)$ и $\operatorname{IIII}^{\mathrm{h}}(C, J) \hookrightarrow \mathrm{III}(C, J)$.

Более того,

$$
\operatorname{III}^{\mathrm{h}}(C, J)=\operatorname{III}(C, J) .
$$

Действительно, существуют канонические вложения

$$
\mathscr{O}_{C, v} \hookrightarrow \mathscr{O}_{C, v}^{\mathrm{h}} \hookrightarrow \widehat{\mathscr{O}}_{C, v},
$$

где $\widehat{\mathscr{O}}_{C, v}=\left(\widehat{\mathscr{O}}_{C, v}^{\mathrm{h}}\right)-$ пополнение $\mathscr{O}_{C, v}$ (см. [7, теорема 18.6.6], [11, гл. I, $\S 1$, замечание $1.2 ;$ гл. I, $\S 4$, упражнение 4.9$])$. Пусть $K_{v}^{\mathrm{h}}-$ поле дробей $\mathscr{O}_{C, v}^{\mathrm{h}}$. Тогда $\widehat{K}_{v}^{\mathrm{h}}-$ поле дробей $\left(\widehat{\mathscr{O}}_{C, v}^{\mathrm{h}}\right)=\widehat{\mathscr{O}}_{C, v}$. Следовательно, $\left(\widehat{K}_{v}^{\mathrm{h}}\right)=\widehat{K}_{v}$. Вложение $K_{v}^{\mathrm{h}} \hookrightarrow \widehat{K}_{v}$ индуцирует каноническое вложение

$$
H^{1}\left(\operatorname{Spec}\left(K_{v}^{\mathrm{h}}\right), J\right) \hookrightarrow H^{1}\left(\operatorname{Spec}\left(\widehat{K}_{v}\right), J\right) .
$$

Действительно, элемент $H^{1}\left(\operatorname{Spec}\left(K_{v}^{\mathrm{h}}\right), J\right)$ представлен торсором $P$ над $K_{v}^{\mathrm{h}}$, который продолжается до плоской проективной схемы $\mathscr{P}$ над $\mathscr{O}_{C, v}^{\mathrm{h}}$. Если $P\left(\widehat{K}_{v}\right) \neq \varnothing$, то $P\left(\widehat{K}_{v}\right)=\mathscr{P}\left(\widehat{\mathscr{O}}_{C, v}\right) \neq \varnothing$, поэтому в силу аппроксимационной теоремы Гринберга $\mathscr{P}\left(\widehat{\mathscr{O}}_{C, v} / \widehat{m}_{v}^{i}\right) \neq \varnothing$ для всех $i[5$, следствие 2$]$; с другой стороны, поскольку $\left(\widehat{\mathscr{O}}_{C, v}^{\mathrm{h}}\right)=\widehat{\mathscr{O}}_{C, v}$, то мы получаем равенство $\mathscr{O}_{C, v}^{\mathrm{h}} /\left(m_{v}^{\mathrm{h}}\right)^{i}=\widehat{\mathscr{O}}_{C, v} / \widehat{m}_{v}^{i}$ для всех $i[1$, гл. III, $\S 2$, п. 12 , изоморфизм (21)]; следовательно, $\mathscr{P}\left(\mathscr{O}_{C, v}^{\mathrm{h}} /\left(m_{v}^{\mathrm{h}}\right)^{i}\right) \neq \varnothing$ для всех $i$, что (по аппроксимационной теореме Гринберга [5, следствие 2]) влечет соотношение $\mathscr{P}\left(\mathscr{O}_{C, v}^{\mathrm{h}}\right) \neq \varnothing$; поэтому $P\left(K_{v}^{\mathrm{h}}\right) \neq \varnothing$ и утверждение $(3.2)$ доказано. Соотношение (3.1) следует из (3.2).

В частности,

$$
\mathrm{III}(C, J) \hookrightarrow \mathrm{III}^{\mathrm{sh}}(C, J) .
$$

3.3. В этом пункте мы доказываем теорему 0.6 .

Пусть $\mathscr{O}_{C, v}^{\mathrm{h}}$ - обычная гензелизация локального кольца $\mathscr{O}_{C, v}$. Это превосходное гензелево дискретно нормированное кольцо с конечным полем вычетов. В силу (3.1) мы имеем

$$
\operatorname{III}^{\mathrm{h}}(C, J)=\operatorname{III}(C, J) .
$$

Максимальное неразветвленное расширение $\left(\mathscr{O}_{C, v}^{\mathrm{h}}\right)^{\text {un }}$ кольца $\mathscr{O}_{C, v}^{\mathrm{h}}$ совпадает со строгой гензелизацией $\mathscr{O}_{C, v}^{\mathrm{h}}[11$, гл. I, $\S 4$, замечание 4.11$]$. В частности, $\left(\mathscr{O}_{C, v}^{\mathrm{h}}\right)^{\mathrm{un}}=$ $\mathscr{O}_{C, v}^{\mathrm{sh}}=\mathscr{O}_{C, \bar{v}}$. Следовательно, $\left(K_{v}^{\mathrm{h}}\right)^{\mathrm{un}}=K_{v}^{\mathrm{sh}}$. Мы обозначаем через $\mathbb{F}_{q_{v}}$ поле вычетов точки $v \in C$. 
Рассмотрим каноническую точную последовательность инфлящии - ограничения $[4$, гл. IV, $§ 5$, предложение 5.1$]$ :

$$
\begin{aligned}
0 & \rightarrow H^{1}\left(\operatorname{Gal}\left(\left(K_{v}^{\mathrm{h}}\right)^{\mathrm{un}} / K_{v}^{\mathrm{h}}\right), J\left(\left(K_{v}^{\mathrm{h}}\right)^{\mathrm{un}}\right)\right) \stackrel{\inf }{\rightarrow} H^{1}\left(\operatorname{Gal}\left(\overline{K_{v}^{\mathrm{h}}} / K_{v}^{\mathrm{h}}\right), J\left(\overline{K_{v}^{\mathrm{h}}}\right)\right) \\
& \stackrel{\text { res }}{\rightarrow} H^{1}\left(\operatorname{Gal}\left(\overline{K_{v}^{\mathrm{h}}} /\left(K_{v}^{\mathrm{h}}\right)^{\mathrm{un}}\right), J\left(\overline{K_{v}^{\mathrm{h}}}\right)\right) .
\end{aligned}
$$

Утверждаем, что существует каноническое вложение

$$
H^{1}\left(\operatorname{Gal}\left(\left(K_{v}^{\mathrm{h}}\right)^{\mathrm{un}} / K_{v}^{\mathrm{h}}\right), J\left(\left(K_{v}^{\mathrm{h}}\right)^{\mathrm{un}}\right)\right) \hookrightarrow H^{1}\left(\operatorname{Gal}\left(\overline{\mathbb{F}_{q_{v}}} / \mathbb{F}_{q_{v}}\right), \pi_{0}\left(J_{v}\right)\left(\overline{\mathbb{F}_{q_{v}}}\right)\right)
$$

где $J_{v}$ - специальный слой минимальной модели Нерона многообразия $J$ в точке $v \in C$ и $\pi_{0}\left(J_{v}\right)=J_{v} / J_{v}^{0}$ - конечная групповая схема над $\mathbb{F}_{q_{v}}$. Этот факт доказан в $[12$, гл. I, $\S 3$, предложение 3.8$]$ для полного локального кольца с конечным полем вычетов. Поскольку доказательство зависит только от леммы Гензеля, оно остается верным для гензелева кольца $\mathscr{O}_{C, v}^{\mathrm{h}}$. Действительно, пусть $\mathscr{A}$ - минимальная модель Нерона многообразия $J \otimes_{K} K_{v}^{\mathrm{h}}$ над $\mathscr{O}_{C, v}^{\mathrm{h}}$. Мы обозначаем через $\mathscr{A}^{0}$ открытую групповую подсхему в $\mathscr{A}$ с общим слоем $J \otimes_{K} K_{v}^{\mathrm{h}}$ и специальным слоемкомпонентой нуля в $J_{v}$. Так как $\mathscr{A}$ гладкая над $\mathscr{O}_{C, v}^{\mathrm{h}}$, то лемма Гензеля показывает, что отображение редукции $\mathscr{A}\left(\left(\mathscr{O}_{C, v}^{\mathrm{h}}\right)^{\mathrm{un}}\right) \rightarrow \mathscr{A}_{v}\left(\overline{\mathbb{F}_{q_{v}}}\right)=J_{v}\left(\overline{\mathbb{F}_{q_{v}}}\right)$ сюръективно (действительно,

$$
\mathscr{A} \times_{\operatorname{Spec}\left(\mathscr{O}_{C, v}^{\mathrm{h}}\right)} \operatorname{Spec}\left(\mathscr{O}_{C, v}^{\mathrm{sh}}\right) \rightarrow \operatorname{Spec}\left(\mathscr{O}_{C, v}^{\mathrm{sh}}\right)
$$

- гладкая схема над строго гензелевым кольцом $\mathscr{O}_{C, v}^{\mathrm{sh}}=\left(\mathscr{O}_{C, v}^{\mathrm{h}}\right)^{\mathrm{un}}$, поэтому отображение редукции

$$
\mathscr{A}\left(\mathscr{O}_{C, v}^{\mathrm{sh}}\right) \rightarrow \mathscr{A}_{v}\left(\overline{\overline{\mathbb{F}} q_{v}}\right)=J_{v}\left(\overline{\mathbb{F}_{q_{v}}}\right)
$$

сюръективно $[11$, гл. I, $\S 4$, упражнение 4.13]). Следовательно, существует точная последовательность

$$
0 \rightarrow \mathscr{A}^{0}\left(\left(\mathscr{O}_{C, v}^{\mathrm{h}}\right)^{\mathrm{un}}\right) \rightarrow \mathscr{A}\left(\left(\mathscr{O}_{C, v}^{\mathrm{h}}\right)^{\mathrm{un}}\right) \rightarrow \pi_{0}\left(J_{v}\right)\left(\overline{\mathbb{F}_{q_{v}}}\right) \rightarrow 0
$$

Более того, $\mathscr{A}\left(\left(\mathscr{O}_{C, v}^{\mathrm{h}}\right)^{\mathrm{un}}\right)=J\left(\left(K_{v}^{\mathrm{h}}\right)^{\mathrm{un}}\right)$, потому что $\mathscr{A} \times_{\operatorname{Spec}\left(\mathscr{O}_{C, v}^{\mathrm{h}}\right)} \operatorname{Spec}\left(\mathscr{O}_{C, v}^{\mathrm{sh}}\right) \rightarrow$ $\operatorname{Spec}\left(\mathscr{O}_{C, v}^{\mathrm{sh}}\right)-$ минимальная модель Нерона многообразия $J \otimes_{K}\left(K_{v}^{\mathrm{h}}\right)^{\mathrm{un}}$. С другой стороны, существует канонический изоморфизм

$$
\operatorname{Gal}\left(\left(K_{v}^{\mathrm{h}}\right)^{\mathrm{un}} / K_{v}^{\mathrm{h}}\right) \simeq \operatorname{Gal}\left(\overline{\mathbb{F}_{q_{v}}} / \mathbb{F}_{q_{v}}\right)
$$

Остается показать, что $H^{1}\left(\operatorname{Gal}\left(\left(K_{v}^{\mathrm{h}}\right)^{\mathrm{un}} / K_{v}^{\mathrm{h}}\right), \mathscr{A}^{0}\left(\left(\mathscr{O}_{C, v}^{\mathrm{h}}\right)^{\mathrm{un}}\right)\right)=(0)$.

Элемент $\alpha \in H^{1}\left(\operatorname{Gal}\left(\left(K_{v}^{\mathrm{h}}\right)^{\mathrm{un}} / K_{v}^{\mathrm{h}}\right), \mathscr{A}^{0}\left(\left(\mathscr{O}_{C, v}^{\mathrm{h}}\right)^{\mathrm{un}}\right)\right)$ может быть представлен $\mathscr{A}^{0}$-торсором $P$. Так как $J_{v}^{0}$ - связная алгебраическая группа над конечным полем, хорошо известная теорема С. Ленга $[14$, гл. VI, $\S 1.4$, следствие 1] показывает, что $J_{v}^{0}$-торсор $P \otimes_{\mathscr{O}_{C, v}^{\mathrm{h}}} \mathbb{F}_{q_{v}}$ тривиален, и поэтому $P_{v}\left(\mathbb{F}_{q_{v}}\right)$ - непустое множество. Лемма Гензеля показывает, что отображение $P\left(\mathscr{O}_{C, v}^{\mathrm{h}}\right) \rightarrow P_{v}\left(\mathbb{F}_{q_{v}}\right)$ сюръективно [11, гл. I, $\S 4$, пример 4.13)], поэтому $P\left(\mathscr{O}_{C, v}^{\mathrm{h}}\right)$ непусто и, значит, $\alpha=0$. Соотношение $(3.5)$ доказано. 
Сушествует такое конечное расширение $\widetilde{\mathbb{F}_{q_{v}}}$ поля $\mathbb{F}_{q_{v}}$, что $\mathrm{Gal}\left(\overline{\overline{\mathbb{F}_{q_{v}}}} \widetilde{\mathbb{F}_{q_{v}}}\right)$ действует тривиально на конечном $\operatorname{Gal}\left(\overline{\mathbb{F}_{q_{v}}} / \mathbb{F}_{q_{v}}\right)$-модуле $\pi_{0}\left(J_{v}\right)\left(\overline{\mathbb{F}_{q_{v}}}\right)$. Рассмотрим каноническую точную последовательность инфлящии - ограничения $[4$, гл. IV, $\S 5$, предложение 5.1]:

$$
\begin{aligned}
0 & \rightarrow H^{1}\left(\operatorname{Gal}\left(\widetilde{\mathbb{F}_{q_{v}}} / \mathbb{F}_{q_{v}}\right), \pi_{0}\left(J_{v}\right)\left(\widetilde{\mathbb{F}_{q_{v}}}\right)\right) \stackrel{\inf }{\rightarrow} H^{1}\left(\operatorname{Gal}\left(\overline{\mathbb{F}_{q_{v}}} / \mathbb{F}_{q_{v}}\right), \pi_{0}\left(J_{v}\right)\left(\overline{\mathbb{F}_{q_{v}}}\right)\right) \\
& \stackrel{\operatorname{res}}{\rightarrow} H^{1}\left(\operatorname{Gal}\left(\overline{\mathbb{F}_{q_{v}}} / \widetilde{\mathbb{F}_{q_{v}}}\right), \pi_{0}\left(J_{v}\right)\left(\overline{\mathbb{F}_{q_{v}}}\right)\right) .
\end{aligned}
$$

Очевидно, что $H^{1}\left(\operatorname{Gal}\left(\overline{\mathbb{F}_{q_{v}}} / \mathbb{F}_{q_{v}}\right), \pi_{0}\left(J_{v}\right)\left(\overline{\mathbb{F}_{q_{v}}}\right)\right)$ - конечная группа, так как $H^{1}\left(\operatorname{Gal}\left(\widetilde{\mathbb{F}_{q_{v}}} / \mathbb{F}_{q_{v}}\right), \pi_{0}\left(J_{v}\right)\left(\widetilde{\mathbb{F}_{q_{v}}}\right)\right)$ - конечная группа [4, гл. IV, $\S 6$, следствие 2$]$ и

$$
\begin{aligned}
H^{1}\left(\operatorname{Gal}\left(\overline{\mathbb{F}_{q_{v}}} / \widetilde{\mathbb{F}_{q_{v}}}\right), \pi_{0}\left(J_{v}\right)\left(\overline{\bar{F}_{q_{v}}}\right)\right) & =\operatorname{Hom}_{\text {cont }}\left(H^{1}\left(\operatorname{Gal}\left(\overline{\mathbb{F}_{q_{v}}} / \widetilde{\mathbb{F}_{q_{v}}}\right), \pi_{0}\left(J_{v}\right)\left(\overline{\mathbb{F}_{q_{v}}}\right)\right)\right) \\
& \simeq \operatorname{Hom}_{\text {cont }}\left(\widehat{\mathbb{Z}}, \pi_{0}\left(J_{v}\right)\left(\overline{\overline{\mathbb{F}_{q_{v}}}}\right)\right)
\end{aligned}
$$

- конечная группа.

Соотношение (3.5) показывает, что $H_{v}=H^{1}\left(\operatorname{Gal}\left(\left(K_{v}^{\mathrm{h}}\right)^{\mathrm{un}} / K_{v}^{\mathrm{h}}\right), J\left(\left(K_{v}^{\mathrm{h}}\right)^{\mathrm{un}}\right)\right)-$ конечная группа.

Очевидно, что $f_{\mathrm{sh}, v}: H^{1}(\operatorname{Spec}(K), J) \rightarrow H^{1}\left(\operatorname{Spec}\left(K_{v}^{\mathrm{sh}}\right), J\right)$ - композиция канонических морфизмов

$$
H^{1}(\operatorname{Spec}(K), J) \stackrel{f_{\mathrm{h}, v}}{\rightarrow} H^{1}\left(\operatorname{Spec}\left(K_{v}^{\mathrm{h}}\right), J\right) \stackrel{\mathrm{res}}{\rightarrow} H^{1}\left(\operatorname{Spec}\left(K_{v}^{\mathrm{sh}}\right), J\right)
$$

потому что $K \hookrightarrow K_{v}^{\mathrm{h}} \hookrightarrow K_{v}^{\mathrm{sh}}$. Мы перепишем (3.4) как

$$
0 \rightarrow H_{v} \stackrel{\inf }{\rightarrow} H^{1}\left(\operatorname{Spec}\left(K_{v}^{\mathrm{h}}\right), J\right) \stackrel{\text { res }}{\rightarrow} H^{1}\left(\operatorname{Spec}\left(K_{v}^{\mathrm{sh}}\right), J\right) .
$$

Рассмотрим коммутативную диаграмму с точными строками и столбцами

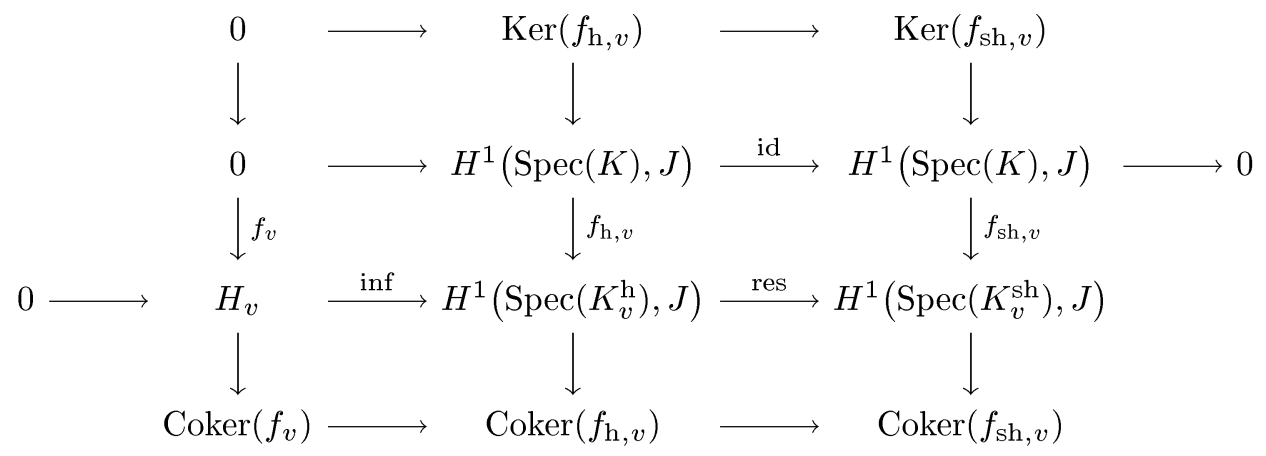

Змеевидная диаграмма $[2$, гл. X, $\S 1.2$, предложение 2$]$ дает точную последовательность

$$
0 \rightarrow \operatorname{Ker}\left(f_{\mathrm{h}, v}\right) \rightarrow \operatorname{Ker}\left(f_{\mathrm{sh}, v}\right) \rightarrow \operatorname{Coker}\left(f_{v}\right)=H_{v} .
$$

Конечность $H_{v}$ показывает, что $\operatorname{Ker}\left(f_{\mathrm{h}, v}\right) \hookrightarrow \operatorname{Ker}\left(f_{\mathrm{sh}, v}\right)$ - подгруппа конечного индекса.

Пусть $A, B, D, F$ - подгруппы в $H^{1}(\operatorname{Spec}(K), J)$. Если $A \hookrightarrow D$ - подгруппа конечного индекса и $B \hookrightarrow F$ - подгруппа конечного индекса, то $A \cap B \hookrightarrow D \cap F-$ подгруппа конечного индекса. 
Действительно, $D / A$ и $F / B$ - конечные группы. Рассмотрим точную последовательность

$$
0 \rightarrow A \rightarrow D \rightarrow D / A \rightarrow 0 \text {. }
$$

Очевидно, что образ $D \cap B$ в $D / A$ - конечная группа, изоморфная $(D \cap B) /(A \cap B)$. С другой стороны, точная последовательность

$$
0 \rightarrow B \rightarrow F \rightarrow F / B \rightarrow 0
$$

показывает, что образ $D \cap F$ в $F / B$ является конечной группой, изоморфной $(D \cap$ $F) /(D \cap B)$. Сушествует точная последовательность

$$
0 \rightarrow(D \cap B) /(A \cap B) \rightarrow(D \cap F) /(A \cap B) \rightarrow(D \cap F) /(D \cap B) \rightarrow 0
$$

Поэтому $(D \cap F) /(A \cap B)$ - конечная группа.

Если $J$ имеет хорошую редукцию в точке $v \in C$, то $\pi_{0}\left(J_{v}\right)=(0)$ и $H_{v}=(0)$; в этом случае (3.6) дает соотношение $\operatorname{Ker}\left(f_{\mathrm{h}, v}\right)=\operatorname{Ker}\left(f_{\mathrm{sh}, v}\right)$. Поскольку $J$ имеет хорошие редукции во всех точках вне конечного подмножества $S \subset C$, то мы видим по замечанию, сделанному выше, что

$$
\operatorname{III}^{\mathrm{h}}(C, J)=\bigcap_{\operatorname{codim}_{C}(v)=1} \operatorname{Ker}\left(f_{\mathrm{h}, v}\right) \hookrightarrow \bigcap_{\operatorname{codim}_{C}(v)=1} \operatorname{Ker}\left(f_{\mathrm{sh}, v}\right)=\operatorname{III}^{\mathrm{sh}}(C, J)
$$

- подгруппа конечного индекса.

Начиная с этого места мы предполагаем, что геометрический слой минимальной модели Нерона многообразия $J$ в любой точке $v \in C$ связен. В этом случае $\pi_{0}\left(J_{v}\right)=(0)$ и $H_{v}=(0) ;$ поэтому (3.7) дает соотношение $\operatorname{Ker}\left(f_{h}\right)=\operatorname{Ker}\left(f_{\mathrm{sh}}\right)$. Следовательно, $\operatorname{III}^{\mathrm{h}}(C, J)=\operatorname{III}^{\mathrm{sh}}(C, J)$. Теорема 0.6 доказана.

3.4. Мы хотим прояснить взаимоотношения между группой Брауэра поверхности $V$ с пучком кривых над $C$, гипотезой Тэйта для $V$ и конечностью $l$-примарных компонент $\mathrm{III}^{\mathrm{sh}}(C, J)$, где $J$ - якобиево многообразие общей кривой пучка.

Доказательство следующей теоремы аналогично доказательству теоремы 4.1 из [19].

3.5. ТеОремА. Пусть $C$ - гладкая проективная кривая над числовым полем $k$, и пусть $f: V \rightarrow C$ - плоский проективный $k$-морфизм $c 1$-мерными слоями. Мы предполагаем, что $V$ и общий слой $f$ гладкие. Предположим, что выполняются следующие условия:

а) существует сечение $\theta: C \rightarrow V$ морфизма $f, C(k) \neq \varnothing$;

b) сечение $\theta$ определяет изоморфизм $\operatorname{Pic}^{0}(C) \simeq \operatorname{Pic}^{0}(V)$.

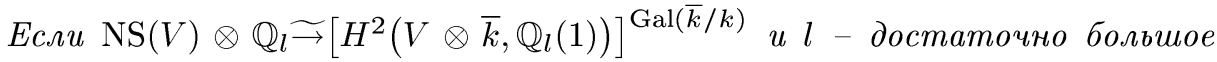
простое число, то l-примарная компонента группь $\operatorname{Br}(V) / \operatorname{Br}(C)$ конечна.

3.6. ЗАмЕчАниЕ. Хорошо известно, что в характеристике нуль пространство модулей гладких проективных кривых рода $g \geqslant 4$ с якобиевой жесткостью уровня $N(N \geqslant 3)$ содержит некоторые проективные кривые, так что для некоторой проективной гладкой кривой $C$ существует неизотривиальное семейство $f: V \rightarrow C$ c гладкими и неприводимыми 1-мерными геометрическими слоями рода $g$. 
3.7. С другой стороны, мы имеем список регулярных поверхностей, удовлетворяющих гипотезе Тэйта: рациональная поверхность, поверхность Энриквеса $[19$, лемма 3.2], поверхность типа К3 [17], поверхность Ферма [19, §3.3].

3.8. В этом пункте мы доказываем теорему 0.7 .

Пусть $K$ - поле рациональных функций на $C$. Сечение $\theta: C \rightarrow V$ дает вложение $H^{3}\left(C, \mathrm{G}_{m}\right) \hookrightarrow H^{3}\left(V, \mathrm{G}_{m}\right)$, так что спектральная последовательность Лере

$$
E_{2}^{p, q}=H^{p}\left(C, R^{q} f_{*} \mathrm{G}_{m}\right) \Longrightarrow H^{p+q}\left(V, \mathrm{G}_{m}\right)
$$

определяет инъективный морфизм $E_{2}^{3,0} \rightarrow E^{3}$. Согласно лемме 1.1 из [19] мы имеем точную последовательность

$$
0 \rightarrow E_{2}^{1,0} \rightarrow E^{1} \rightarrow E_{2}^{0,1} \stackrel{d_{2}^{0,1}}{\rightarrow} E_{2}^{2,0} \rightarrow E_{1}^{2} \rightarrow E_{2}^{1,1} \rightarrow 0
$$

где $E_{1}^{2}=\operatorname{Ker}\left[E^{2} \rightarrow E_{2}^{0,2}\right]$. Поскольку $f$ - строго плоский собственньй морфизм с приведенными и связными геометрическими слоями, то $\mathscr{O}_{C} \simeq f_{*} \mathscr{O}_{V}$ как пучки на $C_{\text {ét }}$, и $f_{*} \mathrm{G}_{m}=\mathrm{G}_{m}[11$, гл. $\mathrm{V}, \S 3.1-3.5]$. Более того, $\theta$ определяет вложение $H^{2}\left(C, \mathrm{G}_{m}\right) \hookrightarrow H^{2}\left(V, \mathrm{G}_{m}\right)$.

Поэтому (3.8) дает точную последовательность

$$
\begin{aligned}
0 & \rightarrow \operatorname{Br}(C)=H^{2}\left(C, \mathrm{G}_{m}\right) \rightarrow \operatorname{Ker}\left[H^{2}\left(V, \mathrm{G}_{m}\right) \rightarrow H^{0}\left(C, R^{2} f_{*} \mathrm{G}_{m}\right)\right] \\
& \rightarrow H^{1}\left(C, R^{1} f_{*} \mathrm{G}_{m}\right) \rightarrow 0 .
\end{aligned}
$$

Следовательно, мы имеем точную последовательность

$$
0 \rightarrow \operatorname{Br}(C) \rightarrow \operatorname{Ker}\left[\operatorname{Br}(V) \rightarrow H^{0}\left(C, R^{2} f_{*} \mathrm{G}_{m}\right)\right] \rightarrow H^{1}\left(C, R^{1} f_{*} \mathrm{G}_{m}\right) \rightarrow 0 .
$$

Очевидно, что $V(k) \neq \varnothing$. По теореме 3.5 мы имеем следуюший результат: для любого простого числа $l \gg 0 l$-примарная компонента группы $\operatorname{Br}(V) / \operatorname{Br}(C)$ конечна. Следовательно, для любого $l \gg 0 \quad l$-примарная компонента групшы кручения $H^{1}\left(C, R^{1} f_{*} \mathrm{G}_{m}\right)$ конечна.

Пусть $i: \eta \hookrightarrow C$ - вложение общей точки $C$, и пусть $B=i_{*}\left(i^{*}\left(R^{1} f_{*} \mathrm{G}_{m}\right)\right)$. Рассмотрим канонический морфизм $R^{1} f_{*} \mathrm{G}_{m} \rightarrow B$ и определим

$$
E=\operatorname{Ker}\left[R^{1} f_{*} \mathrm{G}_{m} \rightarrow B\right], \quad F=\operatorname{Coker}\left[R^{1} f_{*} \mathrm{G}_{m} \rightarrow B\right] .
$$

Слой $R^{1} f_{*} \mathrm{G}_{m}$ в геометрической точке $\bar{v} \rightarrow v \in C$ изоморфен группе $H^{1}\left(V \times_{C} \operatorname{Spec}\left(\mathscr{O}_{C, \bar{v}}\right), \mathrm{G}_{m}\right)$ [11, гл. III, §1, теорема 1.15 , замечание $\left.1.17(\mathrm{a})\right]$, где $\mathscr{O}_{C, \bar{v}}=\mathscr{O}_{C, v}^{\mathrm{sh}}-$ строгая гензелизация локального кольца $\mathscr{O}_{C, v}[11$, гл. I, $\S 4$, замечание 4.11]. Очевидно, что $\operatorname{Spec}\left(\mathscr{O}_{C, \bar{v}}\right)=\operatorname{Spec}\left(\mathscr{O}_{C, v}^{\mathrm{sh}}\right)-$ схема над $\mathscr{O}_{C, v}$.

С другой стороны, $i^{*}\left(R^{1} f_{*} \mathrm{G}_{m}\right)$ - групповая схема локально конечного типа над $\eta$, поэтому слой $B$ в геометрической точке $\bar{v} \rightarrow v \in C$ изоморфен $H^{0}\left(\eta \times_{C} \operatorname{Spec}\left(\mathscr{O}_{C, \bar{v}}\right), i^{*}\left(R^{1} f_{*} \mathrm{G}_{m}\right)\right)$ [11, гл. III, $\S 1$, теорема 1.15 , замечание $1.17(\mathrm{a})]$. Очевидно, что

$$
B_{\bar{v}}=H^{0}\left(\eta \times_{C} \operatorname{Spec}\left(\mathscr{O}_{C, \bar{v}}\right), i^{*}\left(R^{1} f_{*} \mathrm{G}_{m}\right)\right) \simeq H^{1}\left(V_{\eta} \times_{C} \operatorname{Spec}\left(\mathscr{O}_{C, \bar{v}}\right), \mathrm{G}_{m}\right) .
$$


Мы имеем коммутативную диаграмму

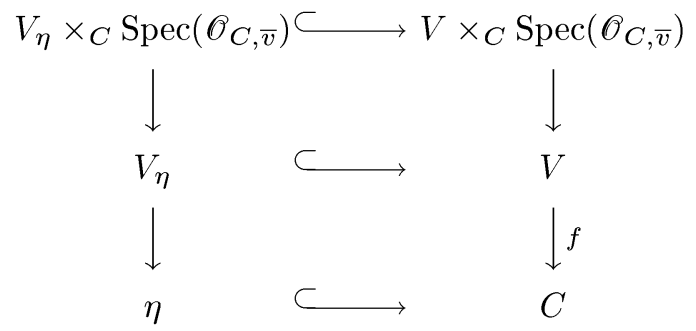

где каждьй прямоугольник дает диаграмму расслоенного произведения.

Напомним, что строгая гензелизация $\mathscr{O}_{C, \bar{v}}$ регулярного локального нётерова кольца $\mathscr{O}_{C, v}$ является регулярным локальным нётеровым кольцом $[7$, предложение 18.8.8, следствие 18.8.13].

Рассмотрим диаграмму расслоенного произведения

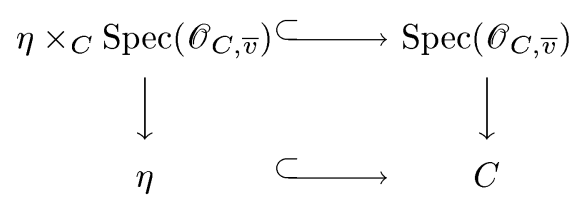

Мы видим из (3.10), что $\eta \times_{C} \operatorname{Spec}\left(\mathscr{O}_{C, \bar{v}}\right)$ - обший слой канонического морфизма $\operatorname{Spec}\left(\mathscr{O}_{C, \bar{v}}\right) \rightarrow C$. С другой стороны, морфизм $\operatorname{Spec}\left(\mathscr{O}_{C, \bar{v}}\right) \rightarrow C$ является композицией канонических морфизмов

$$
\operatorname{Spec}\left(\mathscr{O}_{C, \bar{v}}\right) \rightarrow \operatorname{Spec}\left(\mathscr{O}_{C, v}\right) \hookrightarrow C .
$$

Напомним, что $\mathscr{O}_{C, v}-$ дискретно нормированное кольцо или поле. Следовательно, $\eta \times{ }_{C} \operatorname{Spec}\left(\mathscr{O}_{C, \bar{v}}\right)$ - открытая подсхема в $\operatorname{Spec}\left(\mathscr{O}_{C, \bar{v}}\right)$ и $V_{\eta} \times_{C} \operatorname{Spec}\left(\mathscr{O}_{C, \bar{v}}\right)-$ открытая подсхема в $V \times_{C} \operatorname{Spec}\left(\mathscr{O}_{C, \bar{v}}\right)$.

Очевидно, что $V \times_{C} \operatorname{Spec}\left(\mathscr{O}_{C, v}\right)$ - регулярная схема. С другой стороны, $\mathscr{O}_{C, \bar{v}}-$ объединение этальных конечных расширений $\mathscr{O}_{C, v} \hookrightarrow B_{i}$, и поэтому

$$
\mathscr{O}_{C, \bar{v}}=\lim _{\longrightarrow} B_{i}, \quad \operatorname{Spec}\left(\mathscr{O}_{C, \bar{v}}\right)=\lim _{\longleftarrow} \operatorname{Spec}\left(B_{i}\right) ;
$$

с другой стороны, следующая диаграмма расслоенных произведений

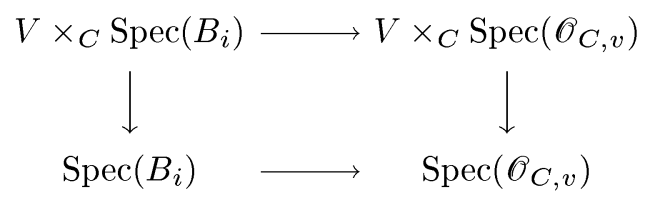

показывает, что $V \times_{C} \operatorname{Spec}\left(B_{i}\right)$ - регулярная нётерова схема, потому что $V \times_{C}$ $\operatorname{Spec}\left(B_{i}\right) \rightarrow V \times_{C} \operatorname{Spec}\left(\mathscr{O}_{C, v}\right)$ - этальный конечный морфизм [11, гл. $1, \S 5$, замечание 5.5]. В частности, для $x \in V \times_{C} \operatorname{Spec}\left(\mathscr{O}_{C, v}\right)$ и для любой такой точки $y_{i} \in V \times_{C} \operatorname{Spec}\left(B_{i}\right)$, что $y_{i}$ отображается в $x$, мы имеем соотношение

$$
m_{y_{i}}=m_{x} \cdot \mathscr{O}_{V \times_{C} \operatorname{Spec}\left(B_{i}\right), y_{i}}
$$


[7, теорема 17.6.1( $\left.\left.c^{\prime}\right)\right]$. Если

$$
y \in \lim _{\longleftarrow} V \times_{C} \operatorname{Spec}\left(B_{i}\right)=V \times_{C} \lim _{\longleftarrow} \operatorname{Spec}\left(B_{i}\right)=V \times_{C} \operatorname{Spec}\left(\mathscr{O}_{C, \bar{v}}\right),
$$

то мы имеем соотношение

$$
m_{y}=m_{x} \cdot \mathscr{O}_{V \times_{C} \operatorname{Spec}\left(\mathscr{O}_{C, \bar{v}}\right), y}
$$

следовательно, $m_{y}$ порождается

$$
\operatorname{dim}\left(\mathscr{O}_{V \times{ }_{C} \operatorname{Spec}\left(\mathscr{O}_{C, \bar{v}}\right), y}\right)=\operatorname{dim}\left(\mathscr{O}_{V \times{ }_{C}} \operatorname{Spec}\left(\mathscr{O}_{C, v}\right), x\right)
$$

элементами, и поэтому $V \times_{C} \operatorname{Spec}\left(\mathscr{O}_{C, \bar{v}}\right)$ - регулярная схема.

Поскольку $V_{\eta} \times{ }_{C} \operatorname{Spec}\left(\mathscr{O}_{C, \bar{v}}\right)$ - открытая подсхема нётеровой регулярной схемы $V \times{ }_{C} \operatorname{Spec}\left(\mathscr{O}_{C, \bar{v}}\right)$, то сушествует каноническая точная последовательность

$$
0 \rightarrow E_{\bar{v}} \rightarrow H^{1}\left(V \times_{C} \operatorname{Spec}\left(\mathscr{O}_{C, \bar{v}}\right), \mathrm{G}_{m}\right) \rightarrow H^{1}\left(V_{\eta} \times_{C} \operatorname{Spec}\left(\mathscr{O}_{C, \bar{v}}\right), \mathrm{G}_{m}\right) \rightarrow 0
$$

[8, гл. II, $\S 6$, предложение 6.5, следствие 6.16]. Поэтому для всех геометрических точек $\bar{v}$ имеем $F_{\bar{v}}=(0)$, и мы получаем точную последовательность

$$
0 \rightarrow E \rightarrow R^{1} f_{*} \mathrm{G}_{m} \rightarrow B \rightarrow 0
$$

Поскольку геометрический слой $f^{-1}(\bar{v})=V_{\bar{v}}$ приведен и неприводим, точная последовательность (3.11) имеет вид

$$
\begin{aligned}
0 & \rightarrow \mathbb{Z} \cdot\left\{\operatorname{\kappa ласc~} f^{-1}(\bar{v}) \text { в } H^{1}\left(V \times_{C} \operatorname{Spec}\left(\mathscr{O}_{C, \bar{v}}\right), \mathrm{G}_{m}\right)\right\} \\
& \rightarrow H^{1}\left(V \times_{C} \operatorname{Spec}\left(\mathscr{O}_{C, \bar{v}}\right), \mathrm{G}_{m}\right) \rightarrow H^{1}\left(V_{\eta} \times_{C} \operatorname{Spec}\left(\mathscr{O}_{C, \bar{v}}\right), \mathrm{G}_{m}\right) \rightarrow 0
\end{aligned}
$$

[8, гл. II, $\S 6$, предложение 6.5, следствие 6.16]. Очевидно, что приведенное неприводимое многообразие $f^{-1}(\bar{v})$ является дивизором $\left(t_{\bar{v}}\right)$ функции $t_{\bar{v}}$, где $t_{\bar{v}}-$ образующая максимального идеала в $\mathscr{O}_{C, \bar{v}}$. Поэтому класс $f^{-1}(\bar{v})$ в $H^{1}\left(V \times_{C}\right.$ $\left.\operatorname{Spec}\left(\mathscr{O}_{C, \bar{v}}\right), \mathrm{G}_{m}\right)$ равен нулю и $E_{\bar{v}}=(0)$. Следовательно,

$$
B=R^{1} f_{*} \mathrm{G}_{m} .
$$

Мы знаем, что для любого $l \gg 0 l$-компонента группы $H^{1}(C, B)$ конечна.

Пусть $J=\operatorname{Pic}^{0}\left(V_{\eta}\right)=B_{\eta}^{0}=\operatorname{Ker}\left[B_{\eta} \stackrel{\text { deg }}{\rightarrow} \mathbb{Z}_{\eta}\right]$ и $A=i_{*}(J)$. Мы получаем из [11, гл. III, $\S 1$, теорема 1.15 , замечание $1.17(\mathrm{a})]$ и (3.9) следующие соотношения:

$$
\begin{gathered}
A_{\bar{v}}=\left(i_{*}(J)\right)_{\bar{v}}=H^{0}\left(\eta \times_{C} \operatorname{Spec}\left(\mathscr{O}_{C, \bar{v}}\right), J\right)=J\left(K_{v}^{\mathrm{sh}}\right)=\operatorname{Pic}^{0}\left(V_{\eta}\right)\left(K_{v}^{\mathrm{sh}}\right), \\
B_{\bar{v}}=H^{0}\left(\eta \times_{C} \operatorname{Spec}\left(\mathscr{O}_{C, \bar{v}}\right), i^{*}\left(R^{1} f_{*} \mathrm{G}_{m}\right)\right)=i^{*}\left(R^{1} f_{*} \mathrm{G}_{m}\right)\left(K_{v}^{\mathrm{sh}}\right)=\operatorname{Pic}\left(V_{\eta}\right)\left(K_{v}^{\mathrm{sh}}\right) .
\end{gathered}
$$

Поэтому $A=\operatorname{Ker}\left[B \stackrel{\operatorname{deg}}{\longrightarrow} \mathbb{Z}_{C}\right]$.

Существование сечения $f: V \rightarrow C$ дает точную последовательность

$$
0 \rightarrow A \rightarrow B \stackrel{\text { deg }}{\rightarrow} \mathbb{Z}_{C} \rightarrow 0 .
$$


Соответствуюшая точная последовательность когомологий имеет вид

$$
\mathbb{Z} \rightarrow H^{1}(C, A) \rightarrow H^{1}(C, B) \rightarrow 0
$$

потому что $H^{1}(C, \mathbb{Z})=(0)$ (действительно, группа этальных когомологий $H^{1}(C, \mathbb{Z})$ является группой кручения [12, гл. II, $\S 2$, лемма 2.10], и поэтому из последовательности когомологий для

$$
0 \rightarrow \mathbb{Z} \stackrel{l}{\rightarrow} \mathbb{Z} \rightarrow \mathbb{Z} / l Z \rightarrow 0
$$

мы получаем точную последовательность $0 \rightarrow H^{1}(C, \mathbb{Z}) \stackrel{l}{\rightarrow} H^{1}(C, \mathbb{Z})$ для всех простых чисел $l$, и, следовательно, $\left.\forall l H^{1}(C, \mathbb{Z})(l)=(0)\right)$.

Поскольку $A=i_{*}(J)$, то спектральная последовательность Лере

$$
E_{2}^{p, q}=H^{p}\left(C, R^{q} i_{*}(J)\right) \Longrightarrow H^{p+q}(\eta, J)
$$

определяет точную последовательность $0 \rightarrow E_{2}^{1,0} \rightarrow E^{1} \rightarrow E_{2}^{0,1}[11$, приложение В]. Она имеет вид $0 \rightarrow H^{1}\left(C, i_{*}(J)\right) \rightarrow H^{1}(\eta, J) \rightarrow H^{0}\left(C, R^{1} i_{*}(J)\right)$; другими словами, мы имеем точную последовательность

$$
0 \rightarrow H^{1}(C, A) \rightarrow H^{1}(\eta, J) \rightarrow H^{0}\left(C, R^{1} i_{*}(J)\right) .
$$

Заметим, что $H^{1}(\eta, J)=H^{1}(\operatorname{Gal}(\overline{k(\eta)} / k(\eta)), J(\overline{k(\eta)}))$ - группа кручения [3, §10, следствие теоремы 10.1], поэтому (3.13) показывает, что $H^{1}(C, A)$ - группа кручения. Следовательно, (3.12) дает точную последовательность

$$
0 \rightarrow \mathbb{Z} / \delta \mathbb{Z} \rightarrow H^{1}(C, A) \rightarrow H^{1}(C, B) \rightarrow 0
$$

где $\delta \in \mathbb{Z}, \delta \neq 0$.

По определению слой $R^{1} i_{*}(J)$ в геометрической точке $\bar{v} \rightarrow v \in C$ равен

$$
\left(R^{1} i_{*}(J)\right)_{\bar{v}}=\lim _{\longrightarrow} H^{0}\left(U, R^{1} i_{*}(J)\right)
$$

для всех коммутативных диаграмм типа

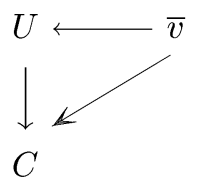

с этальным морфизмом $U \rightarrow C$. Напомним, что в этой ситуации существует каноническое отображение $H^{0}\left(U, R^{1} i_{*}(J)\right) \rightarrow\left(R^{1} i_{*}(J)\right)_{\bar{v}}$, которое обозначается через $s \rightarrow s_{\bar{v}}[11$, гл. II, $\S 2$, замечание $2.9(\mathrm{c})]$.

Для любой точки $v \in C$ выберем геометрическую точку $\bar{v} \rightarrow v \in C$ и рассмотрим каноническое отображение

$$
H^{0}\left(C, R^{1} i_{*}(J)\right) \rightarrow \prod_{v}\left(R^{1} i_{*}(J)\right)_{\bar{v}} \quad\left(s \rightarrow \prod_{v} s_{\bar{v}}\right) .
$$


Предположим, что образ $s \in H^{0}\left(C, R^{1} i_{*}(J)\right)$ равен нулю. По определению $\left(R^{1} i_{*}(J)\right)_{\bar{v}}$ для любой точки $v \in C$ сушествует такой этальный морфизм $U_{v} \rightarrow C$, что его образ содержит $v$ и $\left.s\right|_{U_{v}}=0$. Семейство $\left(U_{v} \rightarrow C\right)$ является покрытием $C$, поэтому $s=0$.

Следовательно, существует вложение

$$
H^{0}\left(C, R^{1} i_{*}(J)\right) \hookrightarrow \prod_{v}\left(R^{1} i_{*}(J)\right)_{\bar{v}}
$$

С другой стороны, $\left(R^{1} i_{*}(J)\right)_{\bar{v}}$ изоморфно $H^{1}\left(\eta \times_{C} \operatorname{Spec}\left(\mathscr{O}_{C, \bar{v}}\right), J\right)[11$, гл. III, $\S 1$, теорема 1.15 , замечание $1.17(\mathrm{a})]$. Очевидно, что

$$
H^{1}\left(\eta \times_{C} \operatorname{Spec}\left(\mathscr{O}_{C, \bar{v}}\right), J\right)=H^{1}\left(\operatorname{Spec}\left(K_{v}^{\mathrm{sh}}\right), J\right) .
$$

Поэтому (3.15) имеет вид

$$
H^{0}\left(C, R^{1} i_{*}(J)\right) \hookrightarrow \prod_{v} H^{1}\left(\operatorname{Spec}\left(K_{v}^{\mathrm{sh}}\right), J\right)
$$

В силу (3.13) и (3.16) мы имеем точную последовательность

$$
0 \rightarrow H^{1}(C, A) \rightarrow H^{1}(\operatorname{Spec}(K), J) \rightarrow \prod_{v} H^{1}\left(\operatorname{Spec}\left(K_{v}^{\mathrm{sh}}\right), J\right) .
$$

Значит, $H^{1}(C, A)=\mathrm{III}^{\mathrm{sh}}(C, J)$. Поскольку $H^{1}(C, B)(l)$ - конечная группа для $l \gg 0$, то мы получаем из (3.14) конечность группы $\mathrm{III}^{\mathrm{sh}}(C, J)(l)$. Конечность $\mathrm{III}(C, J)(l)$ следует из (3.3). Теорема 0.7 доказана.

3.9. Пусть $V$ - гладкая проективная поверхность над числовым полем $k$. Существует такой пучок Лефшеца $f: V^{\prime} \rightarrow \mathbb{P}_{k}^{1}$ сечений $V$ гиперповерхностями степени $d \geqslant 3$, что выполняются следуюшие условия:

a) $V^{\prime} \rightarrow V$ - раздутие конечного множества точек;

b) плоский морфизм $f: V^{\prime} \rightarrow \mathbb{P}_{k}^{1}$ имеет сечение, его обший слой $V_{\eta}^{\prime}$ является гладкой кривой, любой замкнутый слой геометрически приведен и неприводим с не более чем одной обыкновенной двойной особой точкой $[11$, гл. V, $\S 3$, теорема 3.1 , замечание 3.2].

Если гипотеза Тэйта верна для $V$, то она выполняется для $V^{\prime}$.

3.10. В этом пункте мы доказываем следствие 0.8 .

Сечение $\theta$ морфизма $f$ определяет изоморфизм тривиальных алгебраических групп $(0)=\operatorname{Pic}^{0}\left(\mathbb{P}_{k}^{1}\right) \simeq \operatorname{Pic}^{0}\left(V^{\prime}\right)$, потому что $H^{1}\left(V^{\prime}, \mathscr{O}_{V^{\prime}}\right)=(0)$. Поэтому все следует из теоремы 0.7. 


\section{Список литературы}

1. Бурбаки Н. Коммутативная алгебра. М.: Мир, 1971.

2. Бурбаки Н. Гомологическая алгебра. М.: Наука, 1987.

3. Cassels J. W.S. Diophantine equations with special reference to elliptic curves. Survey article // J. London Math. Soc. 1966. V. 41. P. 193-291.

4. Касселс Дж., Фрелих А. Алгебраическая теория чисел. М.: Мир, 1969.

5. Greenberg $M$. Rational points in Henselian discrete valuation rings // Publ. Math. IHES. 1966. V. 31. P. 59-64.

6. Гриффитс Ф., Харрис Джс. Принципы алгебраической геометрии. М.: Мир, 1982.

7. Grothendieck $A$. Eléments de géométrie algébrique. IV. Étude locale des schémas et des morphismes des schémas // Publ. Math. IHES. 1967. V. 32.

8. Хартсхорн Р. Алгебраическая геометрия. М.: Мир, 1981.

9. Кох Х. Теория Галуа $p$-расширений. М.: Мир, 1973.

10. Milne J. S. On a conjecture of Artin and Tate // Ann. Math. 1975. V. 102. P. 517-533.

11. Милн Дж.. Этальные когомологии. М.: Мир, 1983.

12. Milne J. S. Arithmetic duality theorems. N.Y.: Academic Press, Inc., 1986.

13. Мамфорд Д. Лекции о кривых на алгебраической поверхности. М.: Мир, 1968.

14. Серр ЖК. -П. Алгебраические группы и поля классов. М.: Мир, 1968.

15. Серр Ж. -П. Курс арифметики. М.: Мир, 1972.

16. Танкеев С. Г. Об алгебраических циклах на поверхностях и абелевых многообразиях // Изв. АН СССР. Сер. матем. 1981. Т. 45. № 2. С. 398-434.

17. Танкеев C. Г. Поверхности типа К3 над числовыми полями и $l$-адические представления // Изв. АН СССР. Сер. матем. 1988. Т. 52. №6. С. 1252-1271.

18. Tankeev S. G. On the Brauer group of arithmetic scheme. Preprint / Manuskripte der Forshergruppe Arithmetik, 7. Universität Mannheim-Universität Heidelberg, 1999.

19. Танкеев С. Г. О группе Брауэра // Изв. РАН. Сер. матем. 2000. Т. 64. №4. С. 141-162.

20. Tate J. Algebraic cycles and poles of zeta functions // Arithmetical Algebraic Geometry. N.Y.: Harper and Row, 1965. P. 93-110.

21. Tate J. On the conjectures of Birch and Swinnerton-Dyer and a geometric analog // Séminaire Bourbaki 1965/66. Exposé 306. P. 1-26.

Владимирский государственный университет

Поступило в редакцию

E-mail: tankeev-m2@vpti.vladimir.su 1.II. 2000 